\title{
Osteoblast-derived VEGF regulates osteoblast differentiation and bone formation during bone repair
}

\author{
Kai Hu and Bjorn R. Olsen \\ Department of Developmental Biology, Harvard School of Dental Medicine, Boston, Massachusetts, USA.
}

\begin{abstract}
Osteoblast-derived VEGF is important for bone development and postnatal bone homeostasis. Previous studies have demonstrated that VECF affects bone repair and regeneration; however, the cellular mechanisms by which it works are not fully understood. In this study, we investigated the functions of osteoblast-derived VEGF in healing of a bone defect. The results indicate that osteoblast-derived VEGF plays critical roles at several stages in the repair process. Using transgenic mice with osteoblast-specific deletion of Vegfa, we demonstrated that VEGF promoted macrophage recruitment and angiogenic responses in the inflammation phase, and optimal levels of VEGF were required for coupling of angiogenesis and osteogenesis in areas where repair occurs by intramembranous ossification. VEGF likely functions as a paracrine factor in this process because deletion of Vegfr2 in osteoblastic lineage cells enhanced osteoblastic maturation and mineralization. Furthermore, osteoblast- and hypertrophic chondrocyte-derived VEGF stimulated recruitment of blood vessels and osteoclasts and promoted cartilage resorption at the repair site during the periosteal endochondral ossification stage. Finally, osteoblastderived VECF stimulated osteoclast formation in the final remodeling phase of the repair process. These findings provide a basis for clinical strategies to improve bone regeneration and treat defects in bone healing.
\end{abstract}

\section{Introduction}

Bone repair following injury is usually a rapid and efficient process. However, it can be compromised in certain clinical circumstances. For example, delayed union or nonunion of bone fragments occurs in about $5 \%-10 \%$ of patients with bone fracture (1), and bone regenerative procedures sometimes fail. In-depth studies of cellular and molecular mechanisms of bone healing are required for further improvement of procedures aimed at stimulating bone regeneration. Facture healing, the most common form of bone repair, is characterized by several overlapping stages - namely, an inflammation phase, soft callus phase, cartilage turnover phase (replacement by bony callus), and bone remodeling phase (2). After fracture, a hematoma is formed due to disruption of blood vessels and neutrophils are recruited into the hematoma. This is followed by influx of macrophages to remove dead neutrophils, promote angiogenic responses, and initiate the repair cascade (3). With invasion of blood vessels, mesenchymal osteochondral progenitors migrate to the injury site, where they proliferate and differentiate to either osteoblasts or chondrocytes, depending on the stability of facture fixation and supply of blood vessels (4). Finally, the newly formed woven bone is gradually remodeled to lamellar bone.

As one of the most important growth factors for regulation of vascular development and postnatal angiogenesis $(5,6), \mathrm{VEGF}$ also plays critical roles in bone repair, since angiogenesis and osteogenesis are highly coupled $(7,8)$. In the inflammation phase, VEGF is concentrated in hematoma (9) and contributes to the recruitment of macrophages/monocytes $(10,11)$. In endochon-

Conflict of interest: The authors have declared that no conflict of interest exists Submitted: April 28, 2015; Accepted: November 16, 2015.

Reference information: J Clin Invest. 2016;126(2):509-526. doi:10.1172/JCI82585. dral bone formation during bone repair, VEGF promotes recruitment of osteochondroprogenitor cells, induces cartilage formation, and stimulates cartilage resorption and its replacement by bone $(12,13)$. However, inhibition of VEGF impaired bone healing by disrupting conversion of the cartilaginous callus to bony callus (14). Intramembranous bone formation also depends on VEGF signaling. Blocking the VEGF receptors, VEGFR1 and VEGFR2, with neutralizing antibodies decreased blood vessel formation and bone regeneration (15), while administering exogenous VEGF increased formation of mineralized bone within bone defects $(14,16,17)$. In addition, VEGF regulates maturation and differentiation of osteoclasts and, thus, is necessary for maintenance of normal bone remodeling $(14,18,19)$.

These studies demonstrated that the process of bone repair can be affected by manipulation of VEGF levels, but the mechanisms by which different cell types generate and respond to VEGF in repair situations have not been studied. Osteoblast lineage cells, along with chondrocytes, are considered important sources of VEGF in the bone environment $(20,21)$. During bone development, VEGF generated by osteoblast precursors in the perichondrium of cartilage models of future bones stimulates osteoblast differentiation $(22,23)$, and VEGF produced by hypertrophic chondrocytes serves as a chemotactic factor for migration of these osteoblasts into primary ossification centers together with vascular endothelial cells and osteoclasts $(7,8)$. During postnatal bone homeostasis, osteoblast-derived VEGF acts on adjacent endothelial cells to mediate mitogenic and angiogenic effects and plays an important role in maintaining vascular integrity and bone mass $(24,25)$. In addition, intracellular VEGF in osteoblasts regulates the balance of osteoblast and adipocyte differentiation, and disruption of this balance leads to osteoporosis (24). 
Reduced levels of VEGF were observed in many cell types with age, including mesenchymal progenitors (26-30), and this reduction may be related to decreased bone mass and bone vascularity, possibly leading to osteoporosis $(24,31,32)$. Thus, strategies to target VEGF in bone may be of value in cases of defective bone healing and to promote bone regeneration. However, to what extent osteoblast-derived VEGF has the same positive effect in a bone-repair environment as it has during bone development is currently unclear. Also unknown is the relative importance of autocrine and paracrine VEGF signaling mechanisms in osteoblasts and the degree to which these 2 signaling modes have positive and negative effects on the overall repair process. To fill this gap in our understanding, we studied bone repair in mice with a monocortical defect, consisting of a $0.8-\mathrm{mm}$ hole drilled though a single cortex of the tibia. This repair model recapitulates most steps of fracture healing, with the healing response being equivalent to that of a stabilized fracture, and it has many advantages over other fracture models, such as less infection, high survival rate after surgery, and facilitated histomorphometric analysis (33). Conditional deletion of Vegfa or Vefgr 2 in Osterix-expressing (Osx-expressing) osteoblast precursors revealed that osteoblast-derived VEGF acts as a proinflammatory, angiogenic, and osteogenic growth factor during bone healing. It regulates osteoblastic activity by stimulating crosstalk between endothelial, osteoblastic, and hematopoietic cells in a paracrine manner, and it directly affects osteoblast functions via autocrine mechanisms.

\section{Results}

Osteoblastic lineage cells are the important source of VEGF at the bone-repair site. Histology of the cortical defect healing in WT mice at various time points is shown in Supplemental Figure 1A (supplemental material available online with this article; doi:10.1172/ JCI82585DS1). At postsurgery day 7 (PSD7) and PSD10, trabecular bone forms in the defect hole region (the injury area in the interrupted cortical bone) and wounded marrow (Supplemental Figure 1A). Absence of Safranin O-stained cartilage in these regions indicates that newly formed trabecular bone represents intramembranous ossification (IO), while presence of cartilage in the periosteum $(\mathrm{PO})$ close to the injury hole suggests the occurrence of endochondral bone formation (Supplemental Figure 1B). The Cre-activated reporter ZsGreen $(\mathrm{ZsG})$ was used to trace Osx-expressing osteoblastic lineage cells. Of the cells stained with antibodies against VEGF, $72.6 \% \pm 6.2 \%$ were $\mathrm{ZsG}^{+}$cells in the hole region of repair sites at PSD7, and deletion of Vegfa in osteoblastic cells greatly reduced VEGF production in the defects of Vegfat/fl Osx-Cre/ZsG mice (Figure 1A). In normal cortical bone and trabecular bone, VEGF staining was observed in osteoblasts instead of osteocytes (Figure 1B). At the injury sites, VEGF was detected in cells residing along the surface of the newly formed trabeculae but not cells inside the bone (Figure 1B). These results indicate that osteolineage cells, including osteoprecursor cells, preosteoblasts, and mature osteoblasts, are important generators of VEGF in healing of the cortical defect. In the injury region of VE-cadherinCre/tdTomato mice, which were used to trace endothelial lineage cells, only a few tdTomato ${ }^{+}$cells expressed VEGF at PSD7, while moderate levels of VEGF were observed in tdTomato $^{+}$cells of the metaphyseal vasculature (Supplemental Figure 2, A and B).
To further examine the roles of endothelial cell-derived VEGF in healing of bone defects, cortical defects were made in Vegfat/fl $V E$-cadherin-Cre mice and their littermate controls. $\mu$ CT of samples harvested at PSD7 showed that the volume of newly formed bone in the hole region and wounded marrow was practically the same in Vegfall/fl VE-cadherin-Cre, Vegfa ${ }^{f /+} V E$-cadherin-Cre, and Vegfa $a^{f / f l}$ mice (Supplemental Figure 2, C and D), indicating that the small amount of VEGF produced by endothelial cells has little or no role in healing of the type of bone defects examined in this study.

Impaired intramembranous bone formation in defects in mice with VEGF-deficient osteoblastic cells. At PSD7, numbers of $\mathrm{ZsG}^{+}$ cells expressing VEGF were substantially decreased in the hole region of $V e g f a^{f / f l} \mathrm{Osx}$-Cre/ZsG mice compared with Osx-Cre/ZsG mice (Figure 1A), indicating that VEGF was successfully deleted from osteoblastic cells. $\mu \mathrm{CT}$ was used to quantify the newly formed bone in the defect at this stage. Lateral views of the 3D reconstruction of injured tibia showed less mineralized tissue in defects of VEGF-deficient mutants, Vegfafl/fl Osx-Cre (Vegfa CKO) mice, than of controls (Figure 2A). In Vegfa CKO mice, trabecular bone volume (BV) was decreased in the defect hole region (Figure 2B) as well as in the wounded marrow (Supplemental Figure 3A), and this reduction depended on the extent of Vegfa reduction. In addition, mineralized bone in defects of Vegfa CKO mice showed increased trabecular separation (Tb.Sp) and decreased trabecular number (Tb.N); however, trabecular thickness (Tb.Th)was not significantly altered (Figure 2, C-E, and Supplemental Figure 3, B and C). Collagen accumulation, as revealed by aniline blue and movat pentachrome staining, was decreased while the amount of loose fibrous tissue was increased in the defects of Vegfa CKO mice (Figure 2, F and G, and Supplemental Figure 3D), suggesting a decreased amount of osteoid. Decreased mineralization/collagen ratio in Vegfa CKO mice further indicated delay in osteoid mineralization (Figure $2 \mathrm{H}$ ). In addition, BM stromal cells (BMSCs) and osteoblasts from Vegfa CKO mice exhibited reduced mineralization in culture when compared with cells from Vegfa $a^{f / f l}$ mice (Figure 2, I and J). BMSCs from Vegfa ${ }^{f l f l}$ mice also showed decreased mineralization in culture following treatment with Cre adenovirus compared with GFP adenovirus (Figure 2K). In addition, BMSCs or osteoblastic MC3T3-1E cells failed to respond to exogenous VEGF (Supplemental Figure 4, A and B), which is consistent with previous studies (24), indicating a possible role of intracellular VEGF in mineralization. Based on these data, we conclude that loss of VEGF in osteoblastic lineage cells results in a mineralization defect during bone repair.

Osteoblast-derived VEGF stimulates angiogenesis and osteoblast differentiation at bone-repair site. Total number of bone-forming $\mathrm{ZsG}^{+}$cells was not significantly different in defects of Vegfa $a^{f / f l}$ Osx-Cre/ZsG and littermate control mice at PSD7 (Supplemental Figure 5A). Levels of bone sialoprotein (BSP) and osteocalcin $(\mathrm{OCN})$ - markers of osteoblasts at their middle and mature stages of differentiation, respectively - as well as the number of blood vessels, were greatly decreased in the hole region of Vegfatl/fl Osx-Cre/ $Z s G$ compared with $O s x$-Cre/ZsG mice (Figure 3, A-C). Correlation of blood vessel areas with BSP/OCN levels indicated an association between angiogenesis and osteoblast differentiation (Supplemental Figure 5, B and C). The number of $\mathrm{FSP}^{+}$fibroblasts showed a 2.7-fold increase in defects of $V e g f a^{f l / f l} \mathrm{Osx}$-Cre/ZsG mice 
A

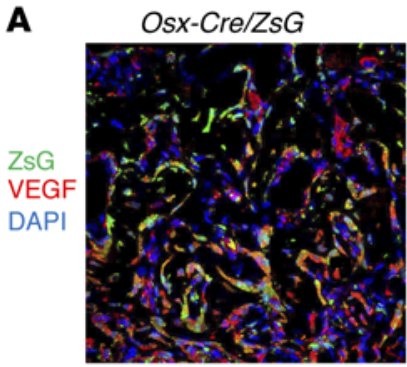

Vegfa" ${ }^{m \prime \prime}$ Osx-Cre/ZsG

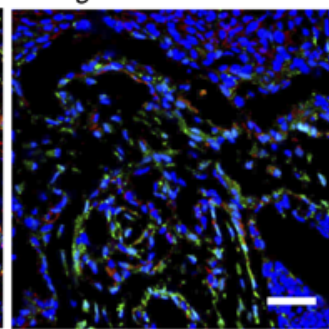

B Trabecular bone under growth plate

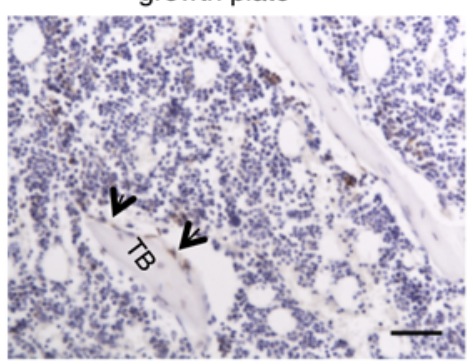

Cortical bone

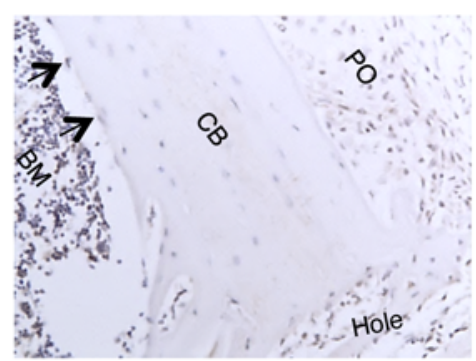

Newly formed bone in hole region

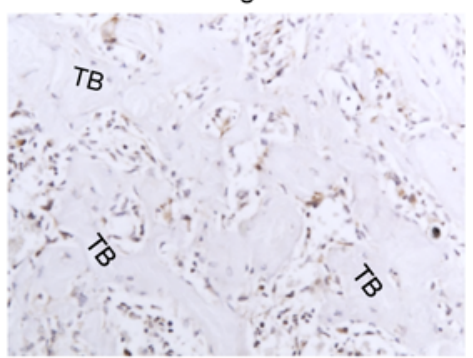

Figure 1. Osteoblastic lineage cells are an important source of VEGF at the bone-repair site. (A) At PSD7, low density (6.1\% $\pm 1.8 \%$ ) of anti-VEGF staining (red) in defect hole region of Vegfa ${ }^{f / f l} \mathrm{Osx}$-Cre/ZsG mice compared with Osx-Cre/ZsG mice $(15.5 \% \pm 2.1 \%) ; P<0.05$. The percentage of VEGF-expressing $\mathrm{ZsG}^{+}$osteolineage cells (yellow) of total ZsG+ cells (yellow cells plus green cells) was $17.0 \% \pm 5.2 \%$ in the hole region of Vegfa ${ }^{f / / f l}$ Osx-Cre/ZsG mice compared with Osx-Cre/ZsG mice (51.5\% $\pm 8.3 \%) ; P<0.05$. Finally, the percentage of VEGF-expressing ZsG ${ }^{+}$osteolineage cells (yellow) of total VEGF-expressing cells (yellow cells plus red cells) was also reduced in hole region of Vegfa ${ }^{f / f l}$ Osx-Cre/ZsG mice $(23.0 \% \pm 1.8 \%)$ compared with Osx-Cre/ZsG mice $(72.6 \% \pm 6.2 \%)$; $P<0.05$. (B) VEGF staining of trabecular bone, cortical bone, and the newly formed bone within cortical defects in WT mice at PSD7. Black arrows indicate VEGF-expressing osteoblasts lining the surface of trabecular bone (TB) or endosteum of cortical bone (CB). Representative images from 3 mice. Scale bar: $50 \mu \mathrm{m}$ (A and B). Unpaired 2-tailed Student's $t$ test was used for comparisons of percentages between groups; $n=4-6$ for each genotype.

compared with controls (Figure 3D), consistent with increased loose fibrous tissue, as visualized by movat pentachrome staining. Costaining for FSP1 in $\mathrm{ZsG}^{+}$cells was more frequently observed in Vegfa ${ }^{f l / f l}$ Os $x$-Cre/ZsG than in Os $x$-Cre/ZsG mice $(18.3 \% \pm 3 \%$ vs. $4.8 \% \pm 1.8 \%$, Figure $3 \mathrm{D}$ ), suggesting that a higher percentage of osteolineage cells differentiate into fibroblasts when VEGF levels are reduced. In addition, more mature fibroblasts were observed in BMSCs from Vegfa CKO mice when cultured in mineralization media compared with cells from Vegfa ${ }^{f l / f l}$ mice (Supplemental Figure 5D). The lack of significant differences in the numbers of tartrate-resistant acid phosphatase-positive $\left(\mathrm{TRAP}^{+}\right), \mathrm{BrdU}^{+}$, and TUNEL $^{+}$cells between the different genotypes (Supplemental Figure 5, E-G) suggested that the impaired repair in Vegfa CKO mice at PSD7 is unlikely to be caused by alterations in osteoclast infiltration, cell proliferation, or cell death.

Postnatal deletion of Vegfa in osteolineage cells impairs IO at the bone-repair site. It can be argued that the compromised bone repair in Vegfa CKO mice might not be a direct consequence of disrupted VEGF production by Osx-expressing cells, but instead the result of VEGF-dependent changes in osteolineage cells during development that are carried over into the postnatal period in mice. To address this possibility and allow assessment of bone repair when VEGF expression is disrupted in the osteoblastic lineage postnatally, doxycycline was administrated to a group of pregnant mothers and their offspring to block Osx-Cre expression (see Methods). Doxycycline was then withdrawn to let Cre recombinase become active in $\mathrm{Osx}^{+}$cells. Without doxycycline treatment,
Vegfa CKO mice showed a decrease in body weight and body size compared with Vegfaflfl mice; however, this difference was eliminated in 8-week-old mice continuously treated with doxycycline (Figure 4A). In addition, the absence of ZsG signal in hind limbs of $V e g f a^{f l f l}$ Osx-Cre/ZsG mice given doxycycline validated the efficient inhibition of Cre expression, while strong ZsG signals in bones of 8-week-old Vegfafl/fl Osx-Cre/ZsG mice with doxycycline withdrawn at 4 weeks demonstrated that Cre recombinase was successfully induced in the absence of doxycycline (Figure 4B). When the bone defect was generated in 9-week-old mice with doxycycline withdrawn at 4 weeks, the volume of newly mineralized bone in the defect hole region of Vegfa CKO mice did not differ from that of the controls (data not shown), suggesting that VEGF produced by $\mathrm{Osx}^{+}$osteoblast precursors and their descendants during embryonic development and the first postnatal 4 weeks could be sufficient to ensure normal bone repair at postnatal week 9. In contrast, in 9-week-old mice with doxycycline withdrawn at 1 week, the volume of newly formed mineralized bone in the defect hole region was decreased in Vegfa CKO mice compared with controls (Figure 4C). For the bone formed in the defect hole region of Vegfa CKO mice, Tb.N was decreased while Tb.Sp was increased (Figure 4C). Collagen accumulation and mineralization/collagen ratio were also decreased (Figure 4D). These data are almost identical to the findings based on the use of mice that had not been treated with doxycycline during development.

Recombinant VEGF affects intramembranous bone formation in cortical bone defects in a dose-dependent manner. Previous 
A
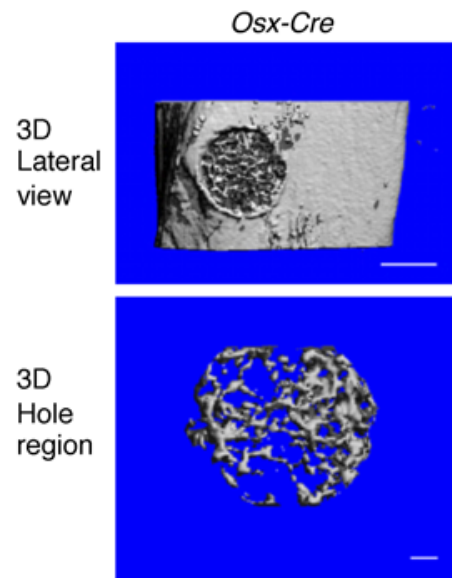

B

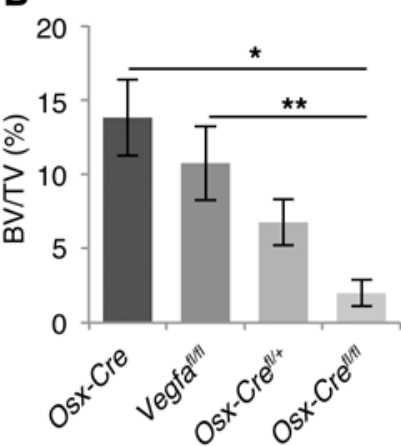

C

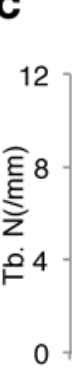


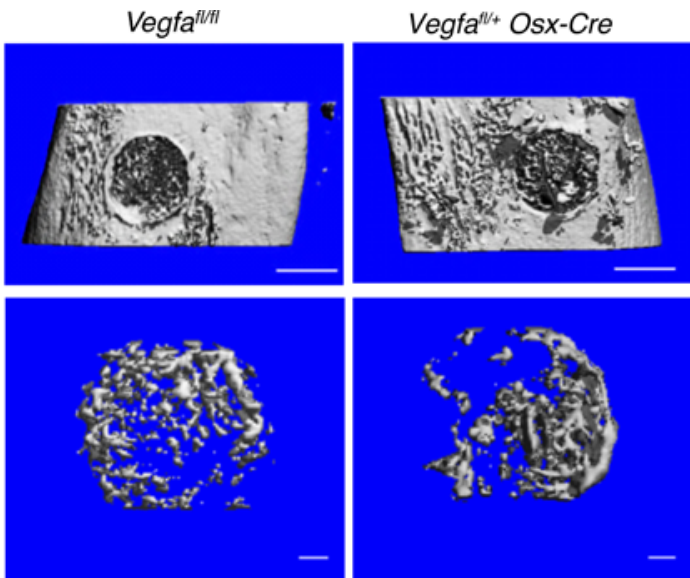

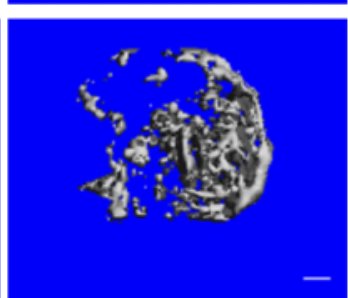

D

D

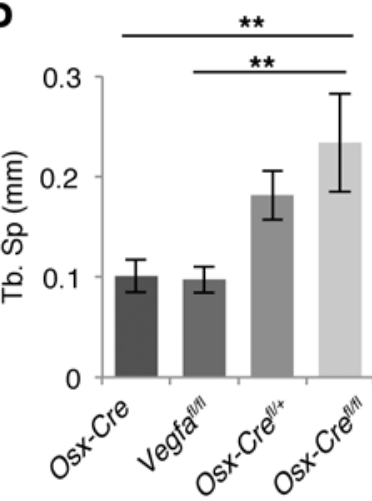

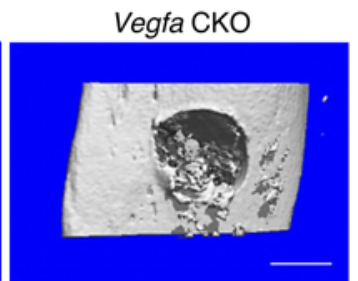



E





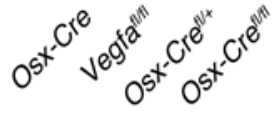

F

Osx-Cre

Vegfa ${ }^{m / n}$

Vegfa $^{\text {ll+ }}$ Osx-Cre

Vegfa CKO

Aniline

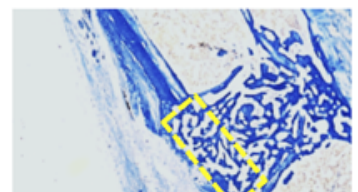

blue
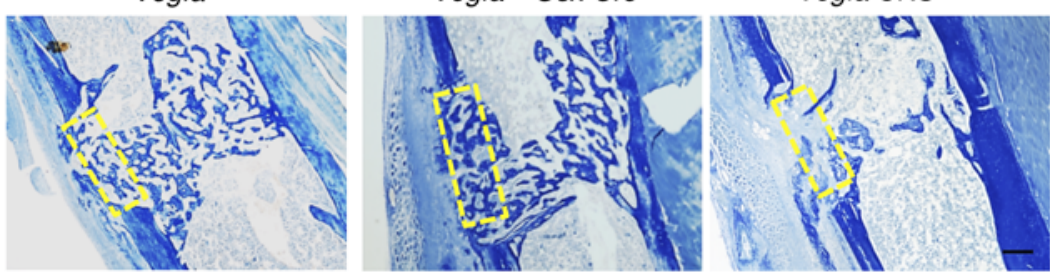

G



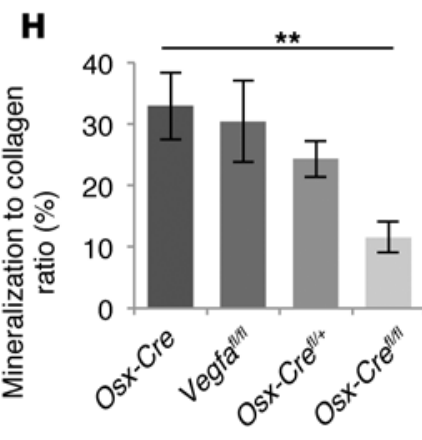

I

BMSCs Vegfattlt Vegfa CKO

Alizarin

Red

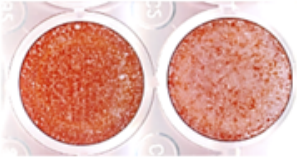

Vegfatt/l Vegfa CKO

Von

Kossa

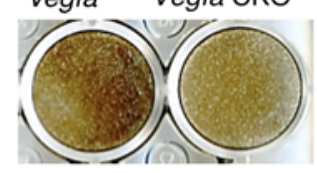

J

Osteoblasts Von

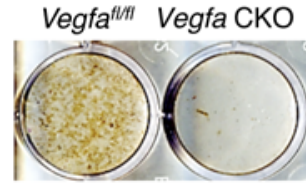

K

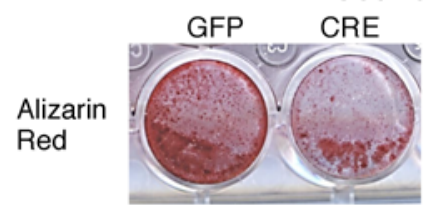

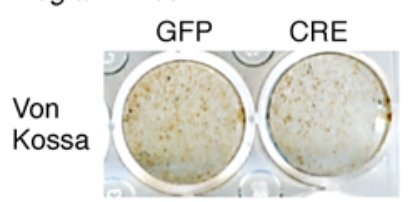


Figure 2. Impaired intramembranous bone formation in defects of Vegfa CKO mice at PSD7. (A) Lateral views of 3D reconstruction of injured tibiae (top panel, scale bar: $500 \mu \mathrm{m}$ ) and mineralized bone formed in hole region (lower panel, scale bars: $100 \mu \mathrm{m}$ ) by $\mu \mathrm{CT}$. Representative images from 6 mice of each genotype. (B-E) 3D structural parameters - trabecular BV/TV\%, Tb.N, Tb.Sp, and Tb.Th - of mineralized bone formed in hole region by $\mu$ CT; $n=6$. (F) Aniline blue staining shows reduced collagen accumulation in hole region of Vegfa CKO mice. Density of aniline blue staining calculated for hole region (stippled yellow rectangles). Scale bar: $200 \mu \mathrm{m}$. (G) Low density of aniline blue-stained area in hole region of Vegfa CKO mice; $n=8-11$. (H) Decreased mineralization/collagen ratio in Vegfa CKO mice; $n=5-6$. (I and J) Alizarin red and Von Kossa staining of BMSCs (I) and osteoblasts (J) after culture in mineralization medium containing $100 \mathrm{nM}$ dexamethasone for 21 days. (K) Alizarin red and Von Kossa staining of BMSCs (from Vegfa ${ }^{f / / f l}$ mice) treated with adenoviral GFP or Cre and cultured in mineralization medium with $100 \mathrm{nM}$ dexamethasone for 21 days. The data are representative of 3 independent experiments (I-K). ANOVA with Tukey's post-hoc test was used. ${ }^{*} P<0.01 ;{ }^{*} P<0.05$.

studies have shown that administration of recombinant VEGF improves compromised bone healing in cortical bone defects $(16,34)$. To determine whether local delivery of recombinant VEGF in a sponge would be able to stimulate the repair process in our model, we first inserted a collagen sponge, loaded with PBS, in the cortical bone defect of WT mice to examine the effects of the sponge on the repair process. When the collagen sponge was applied, the sponge occupied the hematoma space, and the repair process was delayed. Using macrophage recruitment as an indicator of the timing of the inflammatory phase, it appeared that it occurred at PSD5 when the collagen sponge was inserted, compared with PSD3 without the sponge (see below). At PSD7, part of the absorbable sponge loaded with PBS was degraded and mineralized bone was found in the injury region, while maximal induction of mineralized bone formation was observed at PSD10 when most of the sponge was absorbed (data not shown); this suggests that the effects of exogenous VEGF, when loaded into the sponge, may be detected at PSD7 and PSD10 and that most of the VEGF would be released by PDS10. Using a fluorescently labeled VEGF to trace VEGF release in vivo, we found that the majority of VEGF in the sponge was released as early as PSD3. Only a small amount of fluorescent VEGF was observed at PSD7 or PSD10 (Supplemental Figure 6A), consistent with the degradation of the sponge at these time points.

We first studied the effects of $1 \mu \mathrm{g}$ VEGF administration (in $1 \mu \mathrm{l}$ volume), a concentration close to what was used in previous studies $(16,34)$, on membranous bone repair in Osx-Cre/ZsG and Vegfafl/li Osx-Cre/ZsG mice at PSD7. Surprisingly, Osx-Cre/ $Z s G$ mice administered VEGF had less mineralized bone in the hole region than the PBS control group at PSD7 (Supplemental Figure 6B). Collagen accumulation, BSP levels (with or without normalization to the number of $\mathrm{ZsG}^{+}$cells), and the number of blood vessels were also reduced by the VEGF treatment compared with PBS; however, mineralization/collagen ratio was not altered (Supplemental Figure 6, C-E). A bolus of $1 \mu \mathrm{g}$ VEGF decreased the number of $\mathrm{ZsG}^{+}$osteoblastic cells in the injury hole, but this reduction was proportional to the decrease in the total number of cells (Supplemental Figure 6F). Few osteoclasts were observed in both treatment and control PBS groups (Supplemental Figure $6 \mathrm{G}$ ), indicating that decreased bone formation in defects of WT mice treated with a 1- $\mu$ g dose of locally delivered VEGF was not due to increased numbers of osteoclasts. Delivering $1 \mu \mathrm{g}$ VEGF in the sponge also failed to enhance the formation of mineralized bone in the injury region of Vegfal/th $O s x-C r e / Z s G$ mice compared with the control (PBS), both at PSD7 (data not shown) and at PSD10 (Figure 5, A-C), when the maximal amount of mineralized bone can be detected.
Based on these data, we decided to test the effects of a smaller dose: $0.1 \mu \mathrm{g}$ of VEGF. In Osx-Cre/ZsG control mice, delivery of 0.1 $\mu \mathrm{g}$ VEGF failed to improve bone repair in the defects compared with PBS controls at PSD10 (Figure 5, A-C). In contrast, delivering $0.1 \mu \mathrm{g}$ VEGF enhanced formation of mineralized bone in the injury region of $V e g f a^{f / f l} O s x$-Cre/ZsG mice compared with the control (PBS) group (Figure 5, A-C). However, the volume of newly formed bone in all groups of Vegfal/f Osx-Cre/ZsG mice was still below that of $O s x-C r e / Z s G$ control mice (Figure 5, A-C). These data indicate that local delivery of optimal amounts of VEGF enhances bone repair when VEGF levels are low at the repair site but that exogenous VEGF does not promote bone regeneration when endogenous VEGF levels are normal. In defects of Vegfat/fl Osx-Cre/ZsG mice, delivery of $0.1 \mu \mathrm{g}$ VEGF enhanced collagen accumulation and BSP production (Figure 5, D and E). However, the mineralization/collagen ratio and the total number of $\mathrm{ZsG}^{+}$ bone-forming cells did not change (Figure 5, D and F), indicating that, although $0.1 \mu \mathrm{gVEGF}$ restored induction of osteoid formation, it did not affect the delayed mineralization in Vegfalf/fl Osx-Cre/ZsG mice. The number of blood vessels in the hole region was increased in the $0.1 \mu \mathrm{g}$ VEGF treatment group of Vegfat/fl Osx-Cre/ZsG mice, but the vessel parameters were still lower than in $O s x-C r e / Z s G$ mice (Supplemental Figure 7A). This suggests that a bolus delivery of VEGF may not provide sufficient amounts of VEGF during the entire repair process. Treatments with both $0.1 \mu \mathrm{g}$ and $1 \mu \mathrm{g}$ VEGF increased the number of osteoclasts in the hole defect region of Vegfat ${ }^{f / l} \mathrm{Osx}-\mathrm{Cre} / \mathrm{Zs} \mathrm{G}$ mice at PSD10, but the number of osteoclasts was still much lower than in defects of Osx-Cre/ZsG mice (Supplemental Figure 7B). These results indicate that, although VEGF is important for osteoclastogenesis, recruitment of osteoclasts does not appear to be a major factor in bone repair at this stage.

Osteoblast-derived VEGF stimulates macrophage-related angiogenesis and promotes cell infiltration during the inflammatory phase. PSD1-3 is considered as the inflammatory phase in our model. At PSD3, VEGF levels and blood vessels in the defect hole region, adjacent wounded marrow, and area outside hole region were substantially reduced in Vegfa CKO mice compared with control Vegfa ${ }^{A / f}$ littermates (Figure 6, A and B). Costaining for VEGF and for markers of other cell types indicated that macrophages, but not neutrophils and fibroblasts, also generated VEGF in the hole region and adjacent areas (Supplemental Figure 8A). In Vegfa $\mathrm{CKO}$ mice, the number of $\mathrm{F} 4 / 80^{+}$macrophages was reduced in both hole region and adjacent marrow space (Figure 6C). Strong correlation between the density of blood vessels and macrophage numbers indicated that blood vessel invasion is associated with macrophage recruitment (Figure 6D). Supplement of $0.1 \mu \mathrm{g}$ VEGF in a sponge enhanced the early angiogenic response and 
A
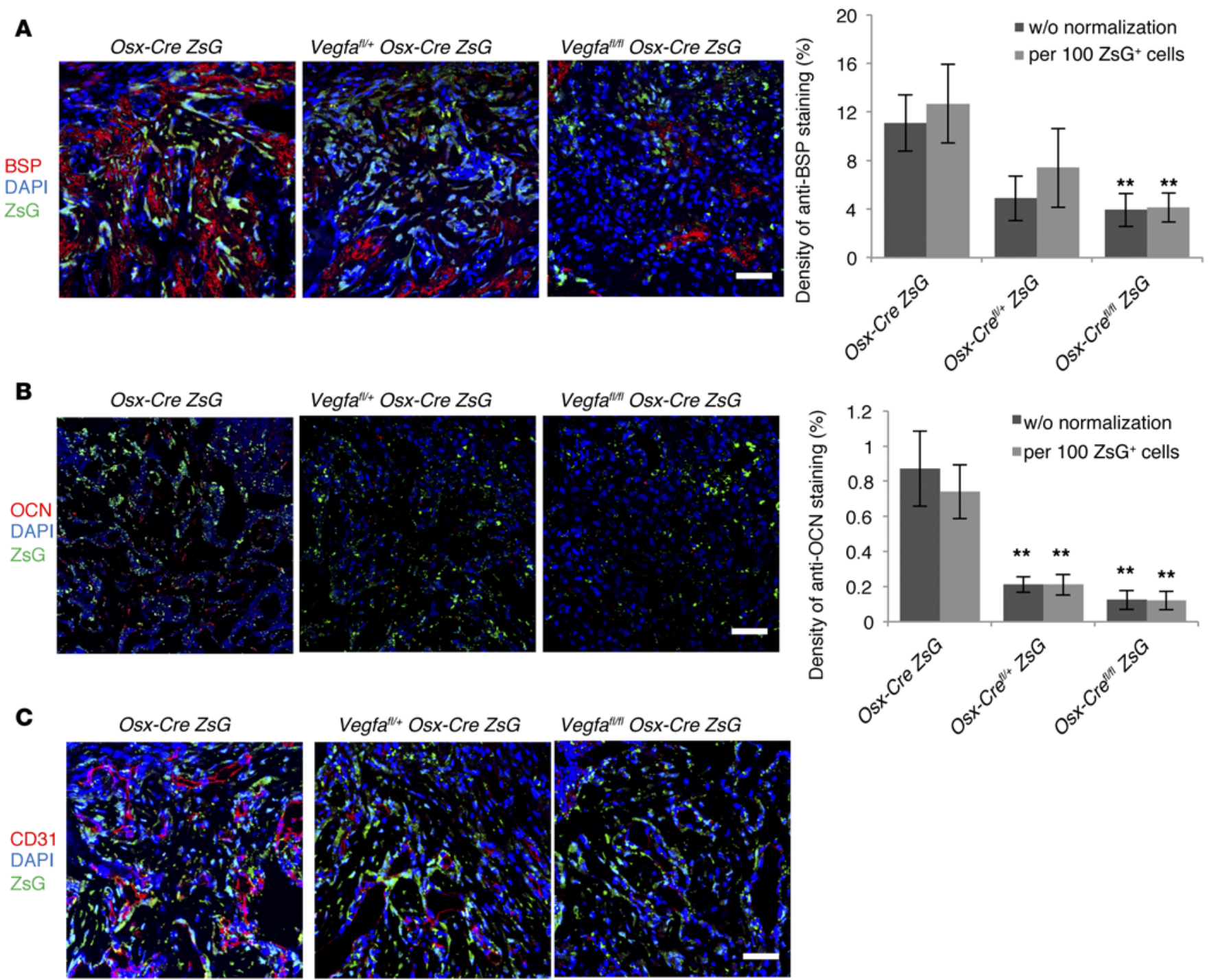

Vegfa"un Osx-Cre ZsG

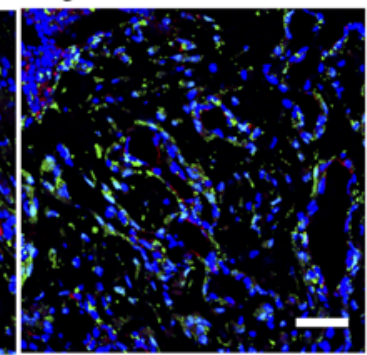

D

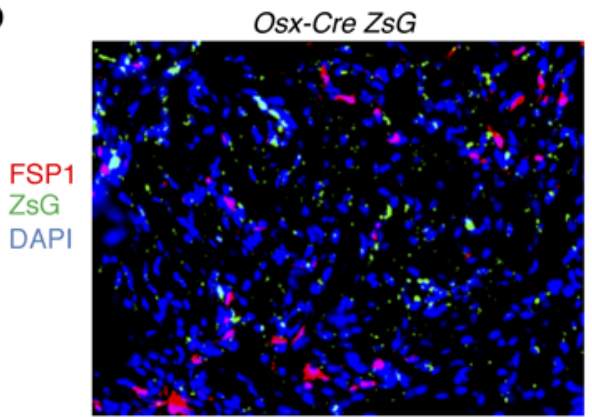

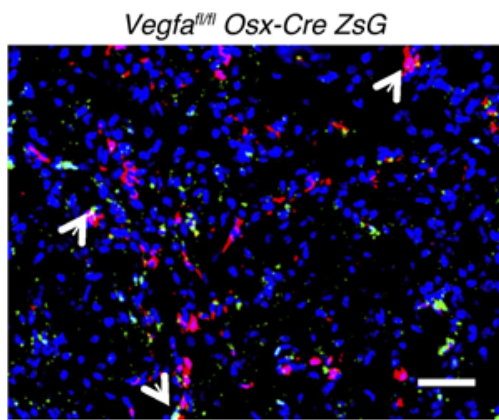

Figure 3. Reduced angiogenesis and osteoblast differentiation in Vegfa ${ }^{f / f l}$ Osx-Cre/ZsG mice at PSD7. (A and B) Low density of anti-BSP-stained (A) or anti-OCN-stained (B) areas with or without normalization to total numbers of ZsG ${ }^{+}$cells, in hole region of Vegfafl/fi Osx-Cre/ZsG mice compared with Osx-Cre/ZsG mice. ${ }^{* *} P<0.05$. (C) Low density $\left(113 / \mathrm{mm}^{2} \pm 34 / \mathrm{mm}^{2}\right)$ of blood vessels (determined by anti-CD31 staining) and low percentage $(4.9 \% \pm 0.7 \%)$ of vessel areas in hole region of Vegfa ${ }^{f / f l}$ Osx-Cre/ZsG compared with Osx-Cre/ZsG mice $\left(227 / \mathrm{mm}^{2} \pm 29 / \mathrm{mm}^{2}\right.$ and $\left.12.6 \% \pm 2.5 \%\right)$; $P<0.05$. Blood vessel density was at an intermediate value $\left(149 / \mathrm{mm}^{2} \pm 23 / \mathrm{mm}^{2}\right)$ in the hole region of Vegfa ${ }^{f l+}$ Osx-Cre/ZsG mice (D) High density

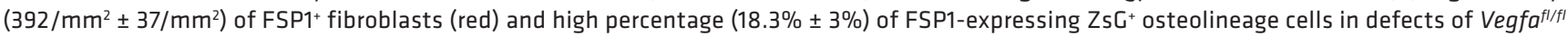
Osx-Cre/ZsG mice compared with Osx-Cre/ZsG mice $\left(189 / \mathrm{mm}^{2} \pm 34 / \mathrm{mm}^{2}\right.$ and $\left.4.8 \% \pm 1.8 \%\right) ; P<0.01$. Yellow cells, indicated by white arrows, represent fibroblasts differentiated from osteolineage cells. Scale bars: $50 \mu \mathrm{m}(\mathbf{A}-\mathbf{D}) ; n=4-6$ for each genotype. Unpaired 2-tailed Student's $t$ test was used for comparisons between the groups. 
A



8-week-old mice with DOX



B

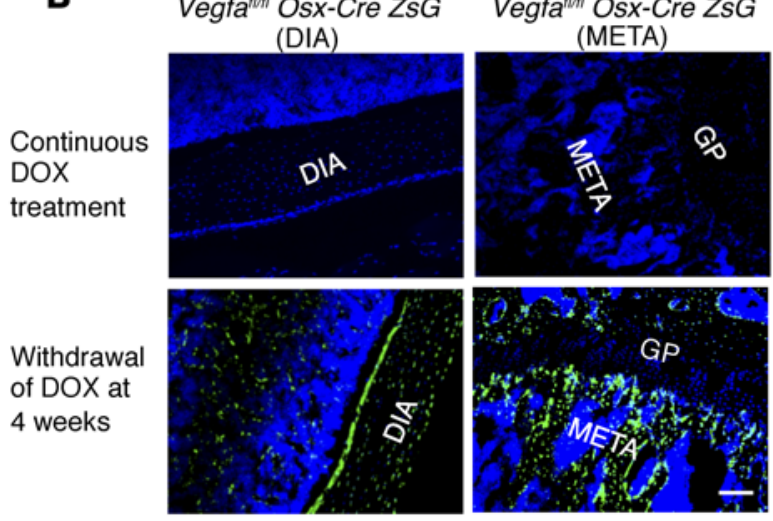



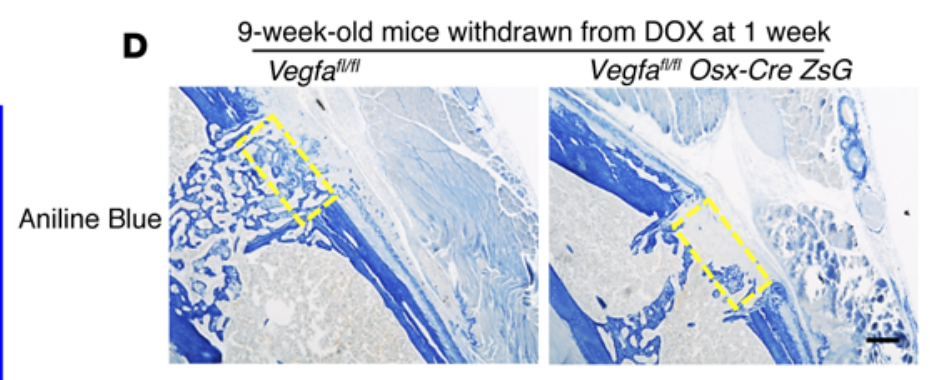

Figure 4. Postnatal deletion of Vegfa in osteoblast lineage cells impairs intramembranous bone formation in cortical defects. (A) Reduced body size and weight in 8-week-old Vegfa CKO mice (18.4 $\pm 0.7 \mathrm{~g})$ compared with Vegfa ${ }^{\text {fl/fl }}$ mice without doxycycline (DOX) treatment (23.4 $\pm 0.7 \mathrm{~g}$ ); $n=8-10, P<0.01$. This reduction was eliminated in DOX-treated mice (20.8 $\pm 0.3 \mathrm{~g}$ and $21.7 \pm 1.1 \mathrm{~g}$ in the $2 \mathrm{groups}) ; n=4-6$. (B) Absence of Creactivated ZsG in diaphysis (DIA) and metaphysis (META) of 8-week-old Vegfa fl/fl Osx-Cre/ZsC mice continuously fed with DOX; ZsG induced in mice by DOX withdrawal at 4 weeks. GP, growth plate. Representative images from 3 mice for each genotype. (C) $\mu$ CT analysis of mineralized bone formed in hole region of 9-week-old Vegfa CKO mice with DOX withdrawn at 1 week shows reduced BV as percentage of total volume (BV/TV, $5.2 \% \pm 1.9 \%$ ), reduced Tb.N $(5.4 / \mathrm{mm} \pm 0.7 / \mathrm{mm})$, but increased Tb.Sp $(0.21 \pm 0.03 \mathrm{~mm})$ compared with that of Vegfaflfl mice $(B V / T V, 19.7 \% \pm 2.4 \% ; \mathrm{Tb} . \mathrm{N}, 11.9 / \mathrm{mm} \pm$ 1.2/mm; Tb. Sp, $0.09 \pm 0.01 \mathrm{~mm}$ ). No significant differences were seen in Tb.Th; $n=6-10$ for each genotype. BV/TV and Tb.N, $P<0.01 ;$ Tb.Sp, $P<0.05$. (D) Reduced density of aniline blue staining $(19.5 \% \pm 4.2 \%)$ and decreased mineralization/collagen ratio $(15.6 \% \pm 5.1 \%)$ in hole region of 9 -week-old Vegfa CKO mice with DOX withdrawn at 1 week, compared with Vegfaflfl mice $(40.8 \% \pm 1.9 \%$ and $43.6 \% \pm 7.2 \%) ; n=6-10$ for each genotype. Aniline blue staining, $P<0.01$; mineralization/collagen ratio, $P<0.05$. Yellow stippled rectangle: hole region. Scale bars: $100 \mu \mathrm{m}$ (B and C), $200 \mu \mathrm{m}$ (D). Unpaired 2-tailed Student's $t$ test was used for comparisons in $\mathbf{C}$ and $\mathbf{D}$.

macrophage recruitment in the defect hole region of Vegfa CKO mice (Figure 6E). Only a few $\mathrm{CD} 45 \mathrm{R}^{+}$cells were recruited into the defects, indicating that lymphocytes were not among the major recruited inflammatory cell types at this stage (Supplemental Figure 8B). The number of $\mathrm{Osx}^{+}$osteoblastic cells was lower in defects of Vegfa CKO mice than of Vegfa $a^{\text {flfl }}$ mice, but this reduction was proportional to the decrease in total cell numbers (Supplemental Figure 8C). Based on percentages of different cellular components in the hole region, only the numbers of macrophages and endothelial cells were lower in Vegfa CKO than in control mice (Supplemental Figure 8D). In addition, in vitro wound closure experiments and transwell migration assay showed that migration of BMSCs from Vegfa CKO mice was reduced compared with cells from Vegfall/fl mice (Figure 6, F and G), suggesting a potential mechanism for the decreased cell infiltration into the repair site in Vegfa CKO mice.

To determine whether loss of osteoblast-derived VEGF affects the release of immune cells into circulation during the acute inflammation phase, peripheral blood collected from mice before and 24 hours after surgery was used for complete blood analysis. The numbers of most blood cells were not significantly different between Vegfa CKO and Vegfalffl mice before surgery, with most parameters in the normal range (Supplemental Figure 9A).
Twenty-four hours after surgery, the number of neutrophils was increased in both Vegfa CKO and controls compared with the number before surgery, indicating the release of neutrophils into the circulation during the acute inflammatory phase (Supplemental Figure 9A). Massive infiltration of NIMP-R14-positive neutrophils was observed in the injury sites, but the number of neutrophils in the hole region was not significantly different between Vegfa CKO and control mice (Supplemental Figure 9B). In addition, only few macrophages were observed in the defects of both Vegfat/lf and Vegfa CKO mice at PSD1 (Supplemental Figure 9C), indicating that influx of macrophages occurs after massive infiltration of neutrophils into the hematoma at PSD1.

Increased cell proliferation and decreased collagen accumulation after injury in mice with VEGF-deficient osteoblastic cells. Following the inflammatory phase, mesenchymal progenitor cells migrate into the wound site, proliferate, and differentiate into osteoblasts. At PSD5, aniline blue staining showed a dramatic reduction of collagen accumulation in the hole region of Vegfa CKO mice (Supplemental Figure 10A). In contrast, BrdU incorporation was increased 2.4-fold in the hole region of Vegfa CKO mice compared with control mice (Supplemental Figure 10B). This enhanced proliferation was associated with an even larger increase in cell 
A

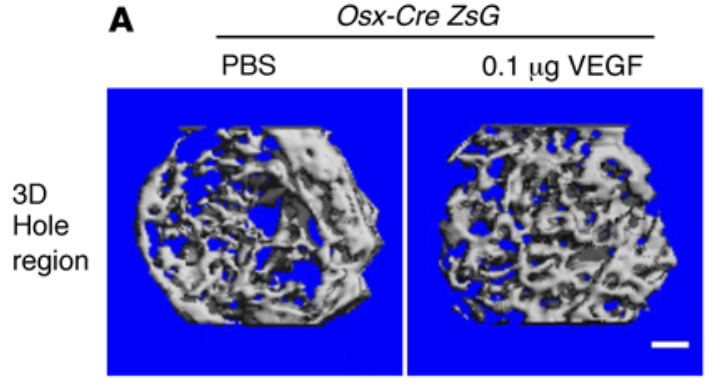

B

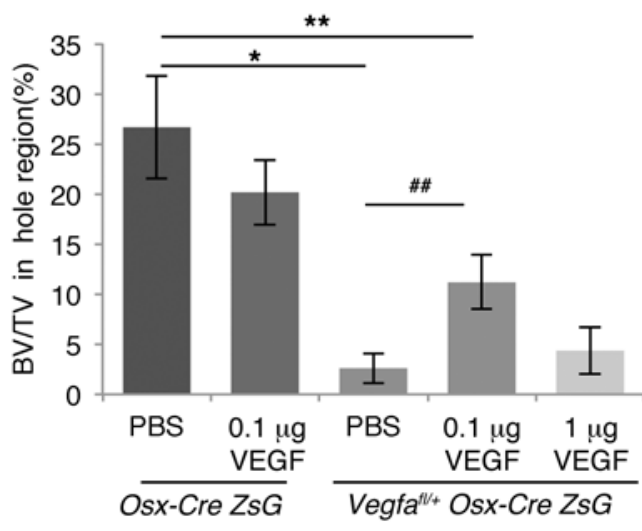

Vegfa $^{f l+}$ Osx-Cre ZsG

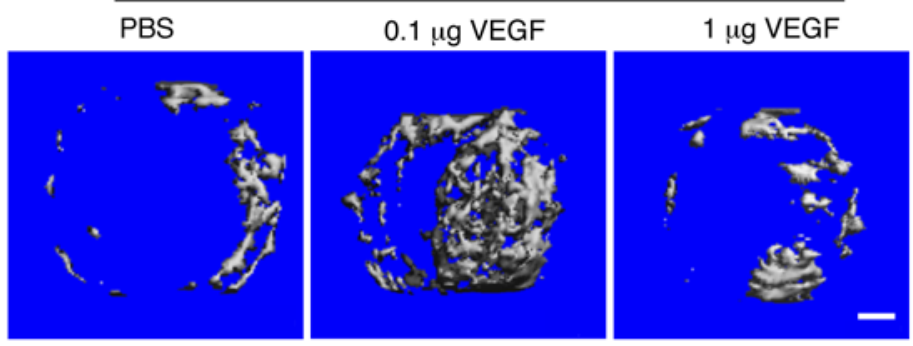

$\star \star$

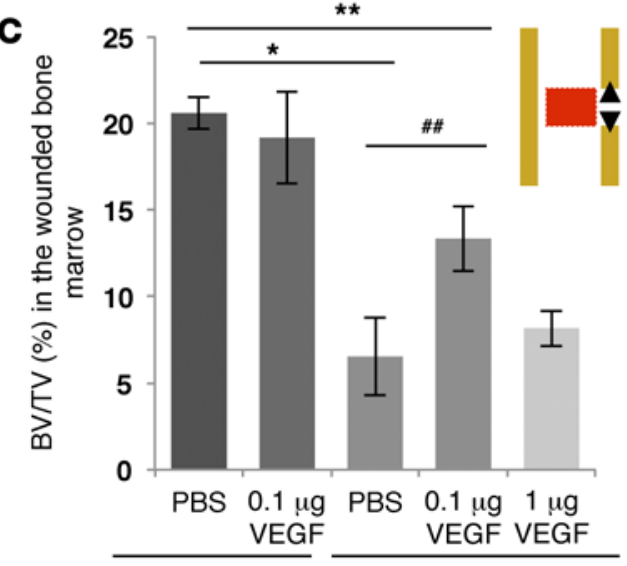

Osx-Cre ZsG Vegfa ${ }^{a /+}$ Osx-Cre ZsG

D


Figure 5. While $0.1 \mu \mathrm{g}$ VEGF enhances, $1 \mu \mathrm{g}$ recombinant VEGF fails to affect intramembranous bone formation in cortical defects of Vegfa fl/fl $O s x$-Cre/ ZsG mice at PSD10. (A) 3D reconstruction of mineralized bone in hole region of Osx-Cre/ZsG mice treated with PBS or $0.1 \mu \mathrm{g}$ VEGF and Vegfafl/fl Osx-Cre/ZsG mice treated with PBS, $0.1 \mu \mathrm{g}$ or $1 \mu \mathrm{g}$ VEGF. (B and C) BV/TV, based on $\mu \mathrm{CT}$ of bone formed in hole region and wounded BM (red area in diagram). (D) Density of aniline blue staining and mineralization/collagen ratio in the hole region (yellow stippled rectangles) of Vegfafl/fl Osx-Cre/ZsG mice treated with PBS (6.3\% $\pm 1.8 \%$ and $47.2 \% \pm 9.0 \%$, respectively) significantly reduced $(P<0.01)$ compared with Osx-Cre/ZsG mice treated with PBS $(24.2 \% \pm 3.2 \%$ and $88.5 \% \pm 7.2 \%$, respectively). Administration of $0.1 \mu \mathrm{g}$ VEGF increases density of aniline blue staining in hole region of Vegfafl/fl Osx-Cre/ZsG mice (18.6\% $\pm 3.5 \%$; $P<0.05$ vs. Vegfafl/fl Osx-Cre/ZsG + PBS), but fails to significantly enhance mineralization/collagen ratio $\left(54.5 \% \pm 10.8 \%\right.$ when compared with Vegfa ${ }^{f / f l}$ Osx-Cre/ZsG mice treated with PBS). Compared with PBS, $1 \mu \mathrm{g}$ VEGF has little effect on aniline blue staining and mineralization/collagen ratio (8.2\% $\pm 1.6 \%$ and $41.6 \% \pm 9.5 \%)$ in Vegfa ${ }^{f / / f l}$ Osx-Cre/ZsG mice. (E) VEGF (0.1 $\left.\mu \mathrm{g}\right)$ enhances anti-BSP staining with or without normalization to ZsG ${ }^{+}$cells in hole region of Vegfa $a^{f / f l}$ Osx-Cre/ZsG mice $(8.9 \% \pm 2.3 \%$ and $7.3 \% \pm 1.4 \%)$ compared with Vegfa $a^{f / f l} O s x-C r e / Z s G$ mice treated with PBS $(1.8 \% \pm 0.6 \%$ and $1.2 \% \pm 0.4 \%) ; P<0.01$ with and $P<0.05$ without normalization. VEGF $(0.1 \mu \mathrm{g})$ has no significant effect $(1.9 \% \pm 0.9 \%$ and $1.0 \% \pm 0.4 \%)$. (F) Density of ZsG $\mathrm{G}^{+}$cells and total cell number in hole region of Vegfa ${ }^{f / f l}$ Osx-Cre/ZsG (CKO) mice not significantly altered by VEGF treatment. Scale bars: $100 \mu \mathrm{m}$ (A), $200 \mu \mathrm{m}$ (D), $50 \mu \mathrm{m}$ (E); $n=5-6$ mice for each group.

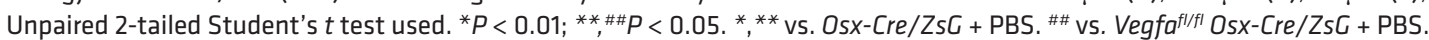


numbers from PSD3-7 in Vegfa CKO compared with control mice (4.5-fold vs. 1.8-fold, Supplemental Figure 10C). In vitro assays showed that recombinant VEGF inhibited, while PDGF-BB greatly induced, proliferation of BMSCs (Supplemental Figure 10, D-F). VEGF also partially inhibited the enhanced proliferation induced by PDGF-BB (Supplemental Figure 10F). These results explain, at least in part, increased proliferation rates at the repair site when VEGF levels were reduced.

Targeting Vegfa expression in osteoblasts and hypertrophic chondrocytes impairs cartilage turnover in periosteal callus. Endochondral bone formation was observed in the injured PO, particularly distal to the injury hole in the tibial defect. Safranin O staining in the PO about $2 \mathrm{~mm}$ distal to the hole was used for quantitative assessments of the cartilage. At PSD7, the relative amount of cartilage in Vegfa CKO mice was similar to that of controls. At PSD10, control mice showed decreased amounts of cartilage, while Vegfa CKO mice showed an increase. At PSD14, there was almost no cartilage left in controls while a substantial amount of cartilage remained in the Vegfa CKO mice (Figure 7, A and B). These data indicate a delay of cartilage removal in PO of Vegfa CKO mice. Compared with Vegfatlfl mice, the numbers of blood vessels and osteoclasts, as well as the amount of mineralized tissues, were significantly reduced in injured PO of Vegfa CKO compared with control mice at PSD10 (Figure 7, $\mathrm{C}-\mathrm{E}$ ), suggesting a delay in blood vessel invasion, osteoclast/ chondroclast recruitment, and endochondral bone formation. We immunostained for collagen type $\mathrm{X}$ to identify hypertrophic chondrocytes and found that the number of hypertrophic chondrocytes was not decreased in PO of Vegfa CKO mice compared with Vegfafl/fl mice at PSD7 (Figure 7F), indicating that decreased mineralization was unlikely due to the delayed maturation of chondrocytes in Vegfa CKO mice. Although the thickness of the periosteal callus was almost the same in Vegfa CKO and Vegfal/fl mice at PSD14, the immature bony tissue in the PO of Vegfa CKO mice as compared with the mature woven bone in Vegfal/fl mice (Supplemental Figure 11A) further suggested delayed endochondral bone formation. Vegfaflfl Osx-Cre/ZsG mice also showed increased amounts of Safranin O-positive cartilage and increased numbers of Sox $9^{+}$chondrocytes in the PO compared with $O s x-C r e / Z s G$ mice at PSD10 (Supplemental Figure 11, B and C). Only few cells coexpressing ZsG and Sox9 were observed, indicating that increased numbers of chondrocytes in Vegfafl/fl Osx-Cre/ZsG mice were not due to transdifferentiation from osteoblastic lineage cells (Supplemental Figure 10C). In addition, lack of significant differences in numbers of $\mathrm{BrdU}^{+}$cells in the injured PO between Osx-Cre/ZsG and Vegfa $a^{f / f l}$ Osx-Cre/ZsG mice (Supplemental Figure 11D), and failure of recombinant VEGF to influence the proliferation of primary chondrocytes in vitro (Supplemental Figure 11E), suggested that increased amounts of cartilage in the injured PO of Vegfa CKO mice were unlikely to be caused by increased chondrocyte proliferation.

Deletion of Vegfa in osteoblastic cells reduces callus remodeling. In WT mice, woven bone formed in the PO was gradually resorbed and remodeled into a new layer of bone outside the original cortical bone. At the edge of the newly formed bone, a new ossification center - containing immature, proliferative, and hypertrophic chondrocytes, as well as bony trabeculae - was observed in periosteal callus of Osx-Cre mice and Vegfat/fl mice at PSD28 (data not shown), reflecting a strong regenerative activity. In contrast, this process was greatly reduced in both homozygous and heterozygous Vegfa conditional KO mice (Figure 8, A and B). The callus thickness (distance between the new layer of bone and the original cortical bone), normalized to the total volume of the corresponding tibial segment, together with volume at callus (also normalized to total tibial segment volume), was reduced in Vegfa CKO mice (Figure 8, C and D). Osteoclasts were accumulated on the surface of the newly formed bony layer in Vegfa $a^{f / f l}$ mice, while only few osteoclasts were found in Vegfa CKO mice (Figure 8E).

Deletion of Vegfr2 in osteoblastic cells increases osteoblast maturation and mineralization in IO-mediated bone repair. To determine whether osteoblast-generated VEGF acts as an autocrine factor via VEGFR2 signaling in osteoblasts, Vegfr2 (Flk1) was conditionally deleted in osteoblastic lineage cells. Based on $\mu \mathrm{CT}$, the amount of mineralized bone within the injury hole was increased in $F l k 1^{l l f l}$ Osx-Cre mice compared with controls at PSD7 (Figure 9, A and B). The Tb.N and Tb.Th were increased in newly formed bone of $F l k 1^{f l f l} \mathrm{Osx}$-Cre mice, while trabecular spacing was decreased (Figure 9A). Although collagen accumulation and BSP levels were not significantly different (Figure 9C and Supplemental Figure 12A), levels of indicators of mineralization, OCN and alkaline phosphatase (ALP), were increased 4.7- and 3.0-fold, respectively, in the defect hole region of Flk $1^{f / f l}$ Osx-Cre mice compared with $F l k 1^{f l / f l}$ mice (Figure 9, D and E). Mineralization/collagen ratio was also greatly increased (Figure 9C). The numbers of blood vessels and $\mathrm{ZsG}^{+}$osteoblastic lineage cells were not different between $F l k 1^{f l / f l} O s x-C r e Z s G$ and control mice, suggesting that increased mineralized bone in the hole region of $F l k 1^{f l f l}$ Osx -Cre mice is not due to increased angiogenesis and numbers of bone-forming cells (Supplemental Figure 12B and Figure 9F). VEGF levels were also similar in the hole region of Flk1 $1^{f l f l}$ Osx-Cre and control mice (Supplemental Figure 12C). Osteoblasts collected from long bones of Flk1 $1^{f / f l}$ Osx-Cre mice showed increased mineralization in mineralization media with or without BMP2 compared with cells from Flk1 $1^{f / f l}$ mice (Figure 9G). In addition, inhibition of FLK1 by the use of a small molecule FLK1 inhibitor enhanced the mineralization of osteoblastic MC3T3-1E cells (Figure 9H), further indicating that osteoblast mineralization is enhanced in $F l k 1^{f / f l}$ Osx-Cre mice. However, BMSCs from Flk $1^{l / f l}$ Osx-Cre mice showed less mineralization than cells from $F l k 1^{f l / f l}$ mice (Supplemental Figure 12D). Treatment with Cre adenovirus decreased the viability of the BMSCs from $\mathrm{Flk}^{\mathrm{fl} / \mathrm{fl}}$ mice compared with GFP-adenovirus treatment (data not shown), indicating that FLK1-mediated signaling is important for the survival of BMSCs. However, TUNEL staining in situ showed no difference in apoptosis between $\mathrm{Flk}^{\mathrm{fl} / \mathrm{fl}} \mathrm{Osx}$-Cre and $F l k 1^{f l / f l}$ samples (Supplemental Figure 12E), indicating that other factors may compensate for loss of FLK1-mediated prosurvival signaling in vivo. There was also no significant difference in periosteal callus thickness and amount of periosteal cartilage in Flk1 ${ }^{f l / f l}$ Osx-Cre and Flk1 $1^{f l / f l}$ mice at PSD10 and PSD14 (Supplemental Figure 13, A and B), indicating that endochondral ossification-mediated periosteal responses were not altered when Flk1 was targeted in osteoblastic cells. 

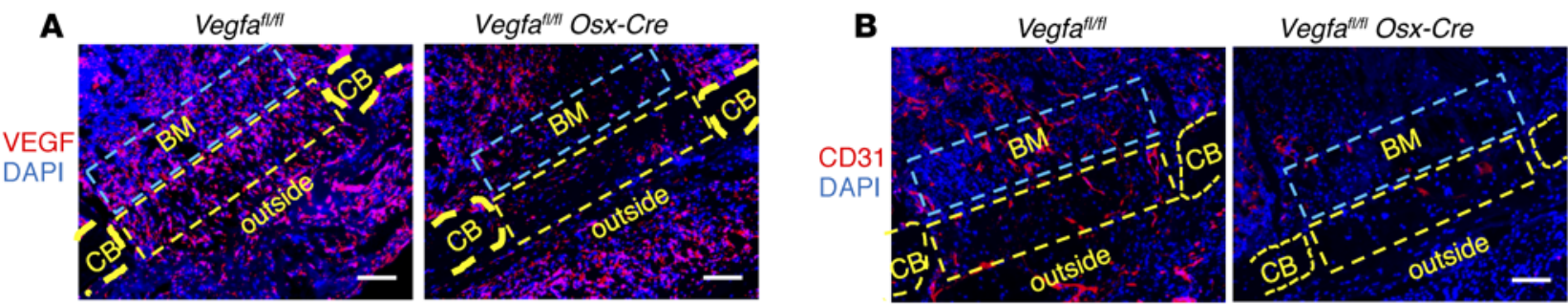

C

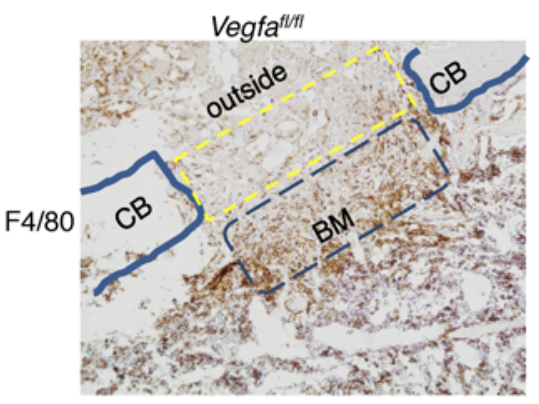

E
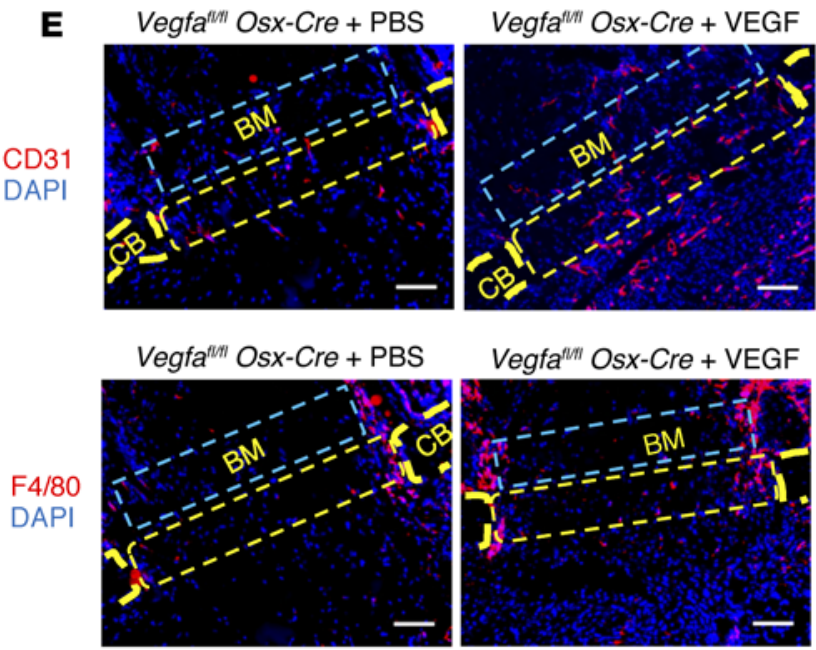

Vegfa $^{f / n}$ Osx-Cre + VEGF



G

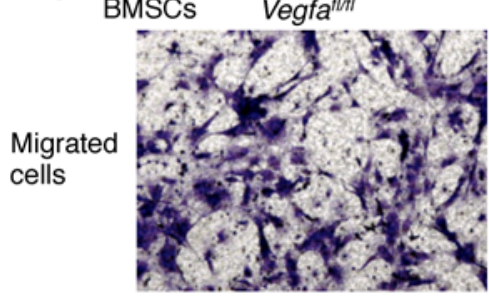

Vegfa" Osx-Cre

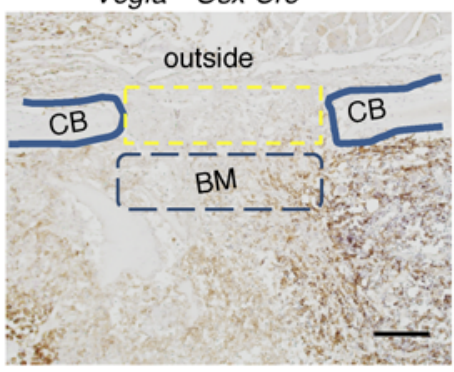

$\mathbf{F}$

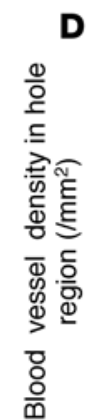

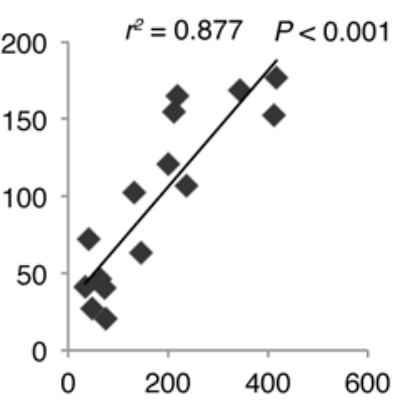

Macrophage density in hole region $\left(/ \mathrm{mm}^{2}\right)$


Figure 6. Osteoblast-derived VEGF stimulates macrophage-related angiogenesis and migration of BM cells. (A) Increased anti-VEGF staining in hole (yellow rectangles) $(17.6 \% \pm 4.8 \%)$ and BM (blue rectangles) $(20.3 \% \pm 4.9 \%)$ of Vegfafl/fl compared with Vegfa CKO mice $(4.2 \% \pm 1.2 \%$ and $6.6 \% \pm 2.0 \%)$ at PSD3; $n=5-6, P<0.05$. No significant differences outside hole (10.2\% $\pm 2.9 \%$ vs. 9.4\% $\pm 2.0 \%)$ (B) High anti-CD31 staining in hole $\left(163 / \mathrm{mm}^{2} \pm 5 / \mathrm{mm}^{2}\right)$, adjacent BM $\left(263 / \mathrm{mm}^{2} \pm 18 / \mathrm{mm}^{2}\right)$, and outside area $\left(164 / \mathrm{mm}^{2} \pm 26 / \mathrm{mm}^{2}\right)$ of Vegfa ${ }^{f / f l}$ compared with Vegfa CKO mice $\left(58 / \mathrm{mm}^{2} \pm 12 / \mathrm{mm}^{2}, 65 / \mathrm{mm}^{2} \pm 21 /\right.$ $\mathrm{mm}^{2}$, and $\left.66 / \mathrm{mm}^{2} \pm 17 / \mathrm{mm}^{2}\right)$ at PSD3; $n=4-7, P<0.05$. (C) High density of $F 4 / 80^{+}$macrophages in hole $\left(321 / \mathrm{mm}^{2} \pm 46 / \mathrm{mm}^{2}\right)$ and $\mathrm{BM}\left(366 / \mathrm{mm}^{2} \pm 69 /\right.$ $\mathrm{mm}^{2}$ ) of Vegfa fl/fl compared with Vegfa CKO mice $\left(82 / \mathrm{mm}^{2} \pm 19 / \mathrm{mm}^{2}\right.$ and $143 / \mathrm{mm}^{2} \pm 26 / \mathrm{mm}^{2}$ ) at PSD3; $n=5-6, P<0.01$ (hole); $P<0.05$ (BM); no difference outside $\left(217 / \mathrm{mm}^{2} \pm 38 / \mathrm{mm}^{2}\right.$ vs. $\left.166 / \mathrm{mm}^{2} \pm 41 / \mathrm{mm}^{2}\right)$. (D) High correlation between blood vessel and macrophage densities in hole. (E) Treating Vegfa CKO mice with $0.1 \mu \mathrm{g}$ VEGF increases blood vessel density in hole $\left(151 / \mathrm{mm}^{2} \pm 7 / \mathrm{mm}^{2}\right)$ and BM $\left(115 / \mathrm{mm}^{2} \pm 9 / \mathrm{mm}^{2}\right)$ and macrophage density in hole $\left(205 / \mathrm{mm}^{2}\right.$ $\left.\pm 34 / \mathrm{mm}^{2}\right)$ at PSD5, compared with PBS $\left(91 / \mathrm{mm}^{2} \pm 12 / \mathrm{mm}^{2}, 50 / \mathrm{mm}^{2} \pm 6 / \mathrm{mm}^{2}\right.$, and $\left.87 / \mathrm{mm}^{2} \pm 9 / \mathrm{mm}^{2}\right) ; P<0.05$. Macrophage density in BM not affected $\left(140 / \mathrm{mm}^{2} \pm 25 / \mathrm{mm}^{2}\right.$ vs. $\left.94 / \mathrm{mm}^{2} \pm 13 / \mathrm{mm}^{2}\right)$. (F) In vitro wound closure; stippled lines indicate wound edges at time 0 . Higher wound closure rate $(\mu \mathrm{m} / \mathrm{h}$ ) in BMSCs of Vegfaflffl $(8.1 \pm 0.7 \mu \mathrm{m} / \mathrm{h})$ than Vegfa CKO mice $(5.1 \pm 0.3 \mu \mathrm{m} / \mathrm{h}) ; n=3, P<0.05$. (G) Transwell migration assay. After 14 hours, more migrated BMSCs from Vegfafl/fl than Vegfa CKO mice; $n=3,{ }^{*} P<0.01$. Scale bars: $50 \mu \mathrm{m}$ (C), $100 \mu \mathrm{m}$ (A, B, and E), $200 \mu \mathrm{m}$ (C and F). CB, cortical bone; outside, area outside cortical bone. Spearman's correlation coefficient test (D) and unpaired 2-tailed Student's $t$ test were used. 


\section{Discussion}

In the present study, we found that appropriate levels of VEGF generated by osteoblastic cells are critical for the angiogenesisosteogenesis coupling during repair of small bone defects. Multiple steps in the repair process require osteoblast-derived VEGF. Either too little or too much VEGF results in compromised bone healing (Supplemental Figure 14). First, osteoblast-derived VEGF is required for the early angiogenic response and macrophage infiltration during the initial inflammation phase. Macrophage infiltration precedes angiogenesis in wound healing and tumor progression $(35,36)$, and VEGF is a chemotactic factor for macrophages/monocytes $(10,11,37)$. Combined with the finding that macrophages release angiogenic factors in the regenerative phase of bone healing (38-40), the evidence presented here suggests that proangiogenic effects of osteoblast-derived VEGF are mediated by macrophages in addition to direct targeting of endothelial cells.

Second, our data demonstrate that osteoblast-derived VEGF stimulates angiogenesis, osteoblast differentiation, and bone formation at the repair site. At the same time, it inhibits chondrogenesis and proliferation of osteoblastic progenitor cells. Consistent with our data are previous studies showing that bone repair and regeneration is inhibited when extracellular VEGF levels are reduced by adding the decoy receptor sVEGFR1 (sFlt1) or VEGF neutralizing antibodies $(14,41)$. However, the rescue experiments with delivery of recombinant VEGF to the defect site in this study indicate that these effects of extracellular VEGF are dose dependent; high levels of VEGF inhibit infiltration of regenerative cells and osteoinduction of mesenchymal progenitors, resulting in decreased bone formation. Since conditional targeting of Flk1 led to increased maturation of osteoblasts and increased formation of mineralized bone at the repair site, we hypothesize that high local concentrations of VEGF stimulate an autocrine FLK1-dependent loop that results in inhibition of osteoblast maturation. Activated FLK1 has been reported to recruit inhibitory $S m a d 7$ and decrease activation of Smad2/3 in endothelial cells (42). This could also be the case in osteoblasts, since osteoblasts collected from $F l k 1^{f l / f l}$ Os $x$-Cre mice exhibited increased mineralization in vitro when stimulated with BMP2, a Smad transducing factor. In addition, VEGF inhibits activation of PDGFR through FLK1 in various cells $(43,44)$. Since PDGF plays an important role in bone formation and regeneration (45), it is also possible that deletion of Flk1 enhances PDGFR signaling in osteoblasts.

These effects may explain why VEGF has been found to enhance bone healing and regeneration in many studies but has failed to have positive effects in others using ex vivo VEGF gene therapy or recombinant VEGF $(13,46-48)$. An important conclusion from the present study is that optimal amounts of VEGF are critical for the therapeutic outcome. The requirement for VEGF depends on the size of the bone wound. In the healing of small bone defects, similar to the one described in the current study, only low concentrations of VEGF may be needed. In this case, adding exogenous VEGF may not stimulate bone regeneration when levels of endogenous VEGF are normal, and adding excess amounts of VEGF may even inhibit osteoblast functions. In larger bone wounds, such as critical or segmental defects, with a greater loss of endothelial cells and osteoblastic progenitors, higher concentrations of VEGF may be needed and inhibitory levels of VEGF may be higher (14). Further highlighting the complexity of VEGF effects in the treatment of bone defects is the finding that osteoinduction was rescued by a low dose of VEGF in Vegfa CKO mice, but osteoblast mineralization was only partially restored. In the case of a relatively small defect, a single bolus addition of VEGF in an absorbable collagen sponge may not be sufficient to cover the need for VEGF throughout the repair process, particularly in the late mineralization stage. VEGF loaded in specific slow-release scaffolds may better stimulate repair in this case $(49,50)$. In addition, failure of exogenous VEGF to fully rescue compromised repair (the mineralization stage) in this study may also be due to the fact that osteoblast-generated VEGF is, in part, intracellular VEGF participating in transcriptional regulation of osteoblast differentiation genes $(24,51,52)$. Future bone-repair studies using modified cell-permeable VEGF, with the capacity to restore intracellular VEGF levels, would therefore be of considerable interest.

Third, the delayed infiltration of blood vessels and osteoclasts into the injured PO and delayed cartilage turnover in the callus of Vegfa CKO mice recapitulates what happens when levels of VEGF are reduced during endochondral ossification in the developing embryo. For example, in mice expressing only the VEGF120 isoform that lacks the ability to bind heparin, and in mice treated with the decoy receptor sVEGFR1 during bone development, endochondral ossification is delayed $(7,23)$. Interestingly, increased periosteal cartilage formation and delayed resorption was observed at the defect of Vegfa CKO mice. This is consistent with studies showing that inhibition of VEGF signaling in early skeletal progenitor cells facilitates a switch in fate from bone to cartilage forming cells (53). In addition, soluble VEGFR1 expression in muscle-derived stem cells in vitro was shown to stimulate BMP4-induced chondrogenic differentiation and promote articular cartilage regeneration $(54,55)$, indicating that blocking VEGF expression or function may improve cartilage healing. Finally, 28 days after the defect was made in control mice, large numbers of osteoclasts residing along the new bone layer in the callus indicated active bone remodeling. In contrast, few osteoclasts were seen in the callus of Vegfa CKO animals. Osteogenic factors released from preosteoclasts or bone matrix after bone resorption - such as TGF- $\beta$, IGF, and PDGF-BB - coordinate formation of blood vessels and bone (56-58). VEGF is also known to stimulate differentiation and activation of osteoclasts $(18,24)$. Therefore, at the remodeling stage, reduction of VEGF in osteoblastic cells may cause decreased osteoclast recruitment. This results in reduced release of osteogenic signals from resorbed bone matrix and subsequent reduction in callus BV.

In clinical studies, associations between VEGF gene polymorphisms regulating VEGF production and spinal bone mineral density or osteoporotic vertebral fractures have been detected in postmenopausal women $(31,59)$. In animal studies, reduced VEGF production was observed in mesenchymal stem cells from aged mice, as well as in the tibial metaphysis, following ovariectomyinduced osteoporosis $(29,32)$. Thus, VEGF supplementation in osteoblasts may be useful in treatment of impaired bone healing as a consequence of age or osteoporosis. Currently, local drug delivery that enhances fracture healing in clinical trials includes BMP2 
A
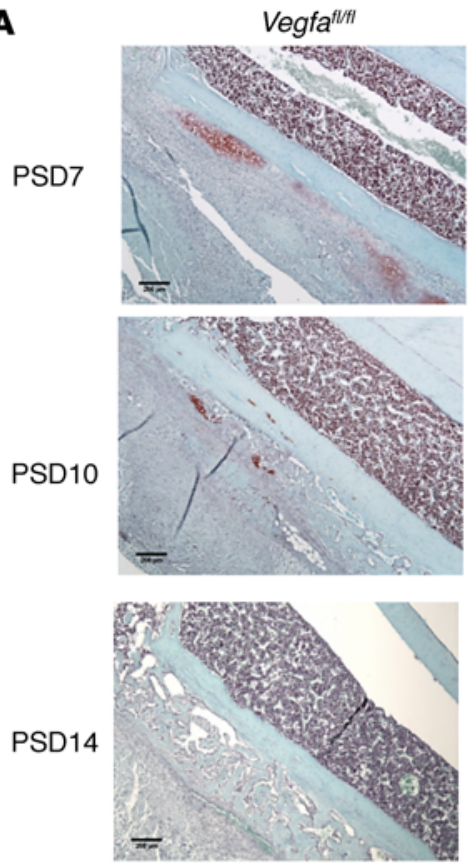

B

E
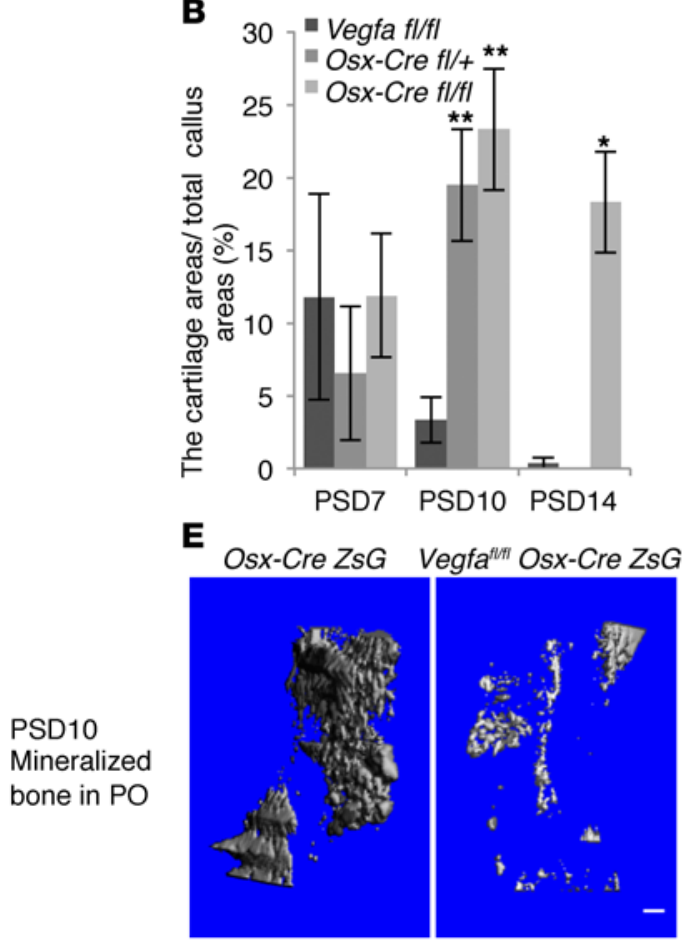

Vegfa ${ }^{f /+}$ Osx-Cre
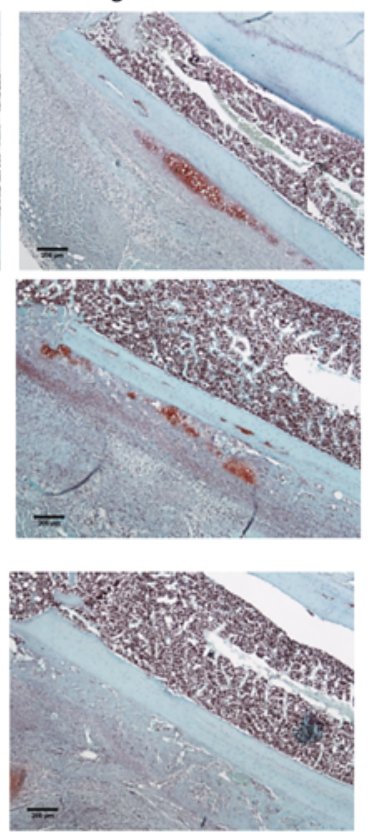

C Vegfatt/t
Vegfat" Osx-Cre
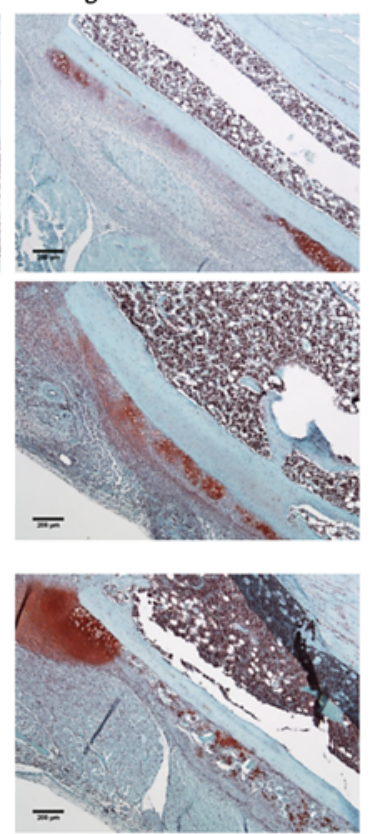

Vegfa $^{\text {"II }}$ Osx-Cre

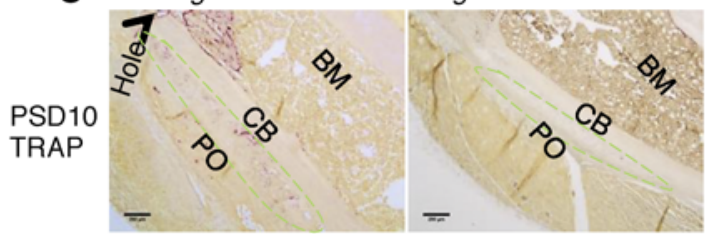

D



F

Vegfa $a^{t \prime \prime \prime}$

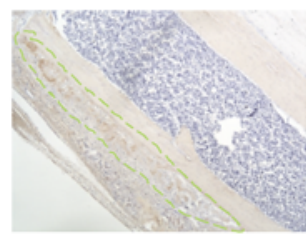

Vegfa" ${ }^{\prime \prime \prime}$ Osx-Cre

PSD7

Col X



Figure 7. Deletion of Vegfa in osteoblasts and hypertrophic chondrocytes impairs cartilage resorption in injured PO. (A) Safranin 0 staining at different time points shows periosteal cartilage in areas about $2 \mathrm{~mm}$ distal to hole region. Representative images from 4-8 mice for each genotype. (B). Periosteal Safranin 0-stained cartilage is gradually resorbed at PSD7-10 in Vegfa ${ }^{f / f l}$ mice; resorption is delayed, and cartilage formation even enhanced in PO of Vegfa CKO mice; $n=4-8,{ }^{*} P<0.01,{ }^{* *} P<0.05$. (C and $\left.\mathbf{D}\right)$ TRAP ${ }^{+}$osteoclasts and lectin ${ }^{+}$vascular endothelial cells greatly decreased in PO of Vegfa CKO mice compared with Vegfafl/fl mice at PSD10. (E) Decreased mineralized BV in PO of Vegfa fl/fl Osx-Cre/ZsG mice $\left(0.0071 \pm 0.0034 \mathrm{~mm}^{3}\right)$ compared with Osx-Cre/ZsG mice $\left(0.069 \pm 0.019 \mathrm{~mm}^{3}\right)$ at PSD10; $n=3-4, P<0.01$. (F) Similar density of collagen X-stained hypertrophic chondrocytes in PO of Vegfa CKO (146/mm² $\pm 21 /$ $\mathrm{mm}^{2}$ ) and Vegfa $a^{f l / f l}$ mice $\left(144 / \mathrm{mm}^{2} \pm 31 / \mathrm{mm}^{2}\right)$ at PSD7; $n=7-9$. Green stippled area represents injured PO. CB, cortical bone. Representative images of 3-4 mice (C-E). Scale bar: $100 \mu \mathrm{m}(\mathbf{E}), 200 \mu \mathrm{m}$ (A, C, D, and F). ANOVA with Tukey's post-hoc test (B) and unpaired 2-tailed Student's $t$ test (E and F) were used. 
A
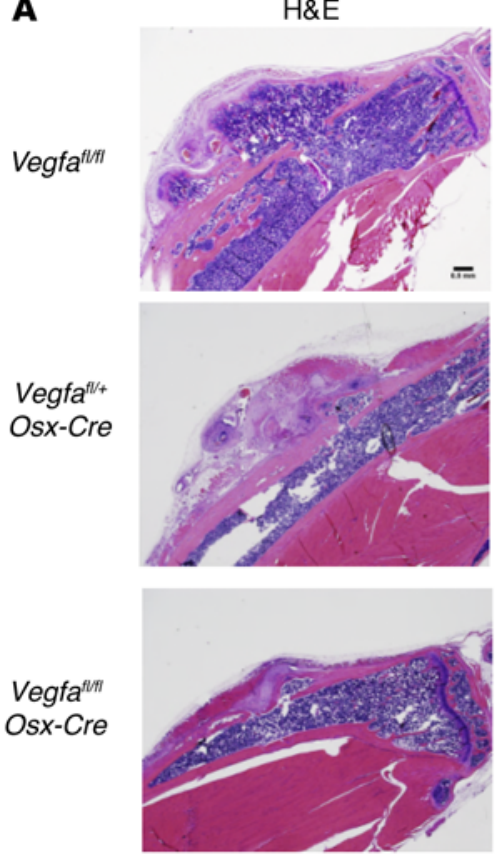

Vegfatth Osx-Cre

\section{C}



E

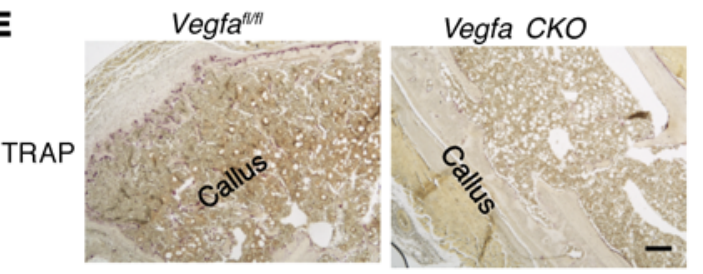

B

$\operatorname{Vegfa}^{n /+}$ Osx-Cre

D


Figure 8. Deletion of Vegfa in osteoblastic cells reduces periosteal callus remodeling at PSD28. (A) H\&E-stained sections from Vegfafl/ft, Vegfa ${ }^{f / /+}$ Osx-Cre, and Vegfa CKO mice showing periosteal callus remodeling at PSD28. Representative images from 5-6 mice for each genotype. (B) Left panels: 2D images of sagittal sections of injured tibiae. Right panels: $3 \mathrm{D}$ reconstruction of periosteal callus. Representative images from 5-6 mice. (C) Decreased callus thickness, calculated as distance from edge of periosteal callus to injured cortical bone normalized to total volume of corresponding tibial segment, in Vegfa CKO compared with Vegfa ${ }^{f / / f l}$ mice; $n=5-6$. (D) Decreased BV/TV, total callus BV normalized to total volume of the corresponding tibial segment, in Vegfa CKO compared with Vegfa ${ }^{f / f l}$ mice; $n=5-6$. (E) Low density of TRAP ${ }^{+}$osteoclasts, normalized to total length of callus bone, in Vegfa CKO compared with Vegfa ${ }^{f l f l}$ mice; $n=4$. Scale bars: $500 \mu \mathrm{m}$ (A and B), 200 $\mu \mathrm{m}(\mathrm{E})$. ANOVA with Tukey's post-hoc test (C and $\mathbf{D}$ ) and unpaired 2-tailed Student's $t$ test $(\mathbf{E})$ were used. ${ }^{*} P<0.01 ;{ }^{* *} P<0.05$

chemical compounds, such as statins, that modulate VEGF expression in osteoblasts should be considered for treatment of osteoporosis and osteoporotic fractures (62-64).

In summary, our data reveal that VEGF, produced by osteoblast lineage cells, is important during various stages of bone defect repair. The new insights into the details of VEGF functions - in an environment rich in growth factors, skeletal stem cells, and bone matrix proteins - may provide a basis for therapeutic strategies based on VEGF modulation to improve bone repair and regeneration. and basic FGF (60). However, therapeutic delivery of VEGF is primarily at the preclinical stage. The optimal dosage, duration of treatment, and delivery mechanisms for VEGF-carrying scaffolds are still under investigation and therefore prevent the transition to clinical trials. Appropriate scaffolds for slow release of physiological amounts of VEGF are needed, and application of exogenous VEGF needs to be carefully considered, especially when evidence for VEGF reduction is lacking in patients. The combination of VEGF with other growth factors, such as TGF- $\beta$ family members, should be taken into consideration, since VEGF may potentially inhibit downstream TGF- $\beta 1$ signaling (42), and high doses of VEGF inhibit BMP2/BMP4-induced bone healing in a critical bone defect model $(13,61)$. Modulation of VEGF levels may be of particular interest in future studies using BMPs to enhance bone repair. In addition to delivery of VEGF to local bone-repair sites,

\section{Methods}

Supplemental Methods are available online with this article.

Mouse strains. Osx-Cre (65), VE-cadherin-Cre (66), CAG-loxP-stoploxP-ZsG (Ai6), and CAG-loxP-stop-loxP-tdTomato (Ai14) mouse strains were purchased from The Jackson Laboratory. Vegfallfl and Flk-1 ${ }^{f / f l}$ mice were provided by Genentech. To generate Vegfa CKO mice, hemizygous Osx-Cre transgenic mice were crossed with Vegfafl/fl mice to produce heterozygous Vegfa $a^{f /+}$ offspring carrying a Cre allele. These mice were then crossed with Vegfal/fll mice generating the following 3 genotypes: Vegfa CKO, Vegfafl/+ Osx-Cre, and Vegfafl/l. For studies of the consequence of postnatal deletion of Vegfa, we took advantage of the presence of a tetracycline responsive element (TRE; tetO) in the Osx-Cre transgene in the Osx-Cre strain to suppress expression of Cre by giving pregnant mice and their progeny water containing $2 \mathrm{mg} / \mathrm{ml}$ doxycycline. The efficiency of Osx-Cre-mediated targeting of Vegfa was assessed in 

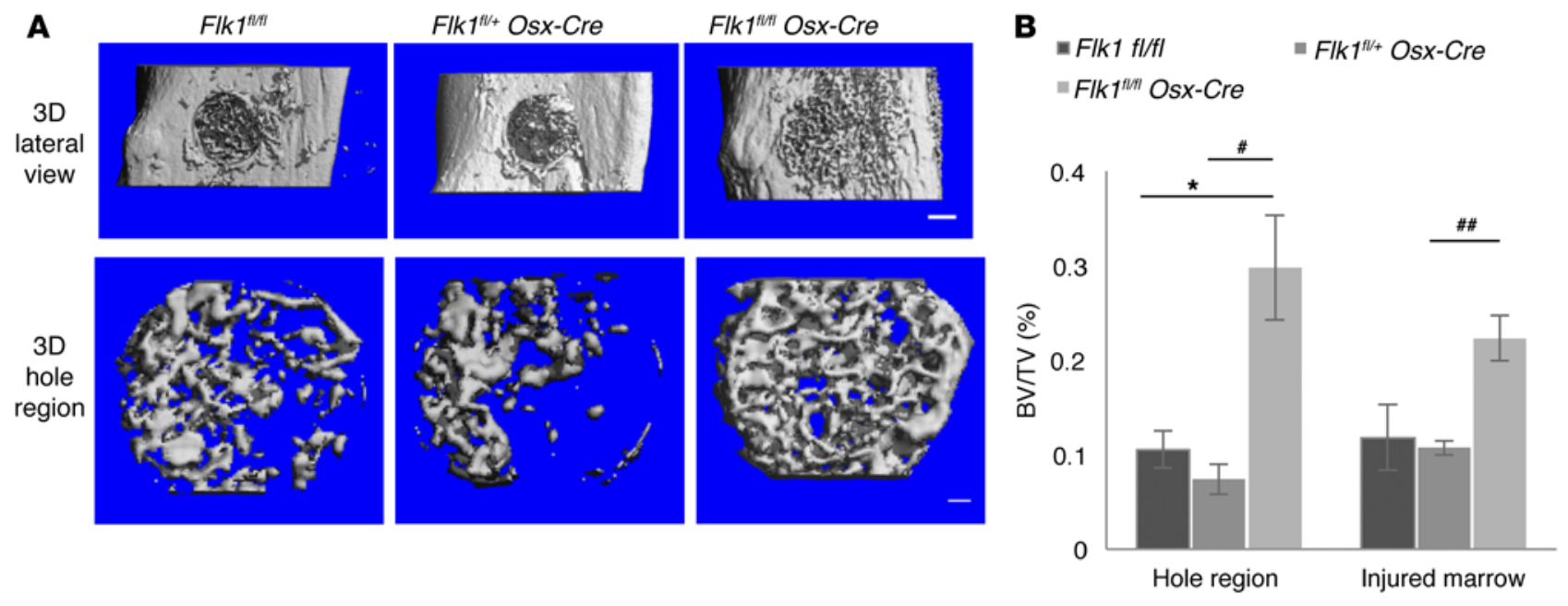

C
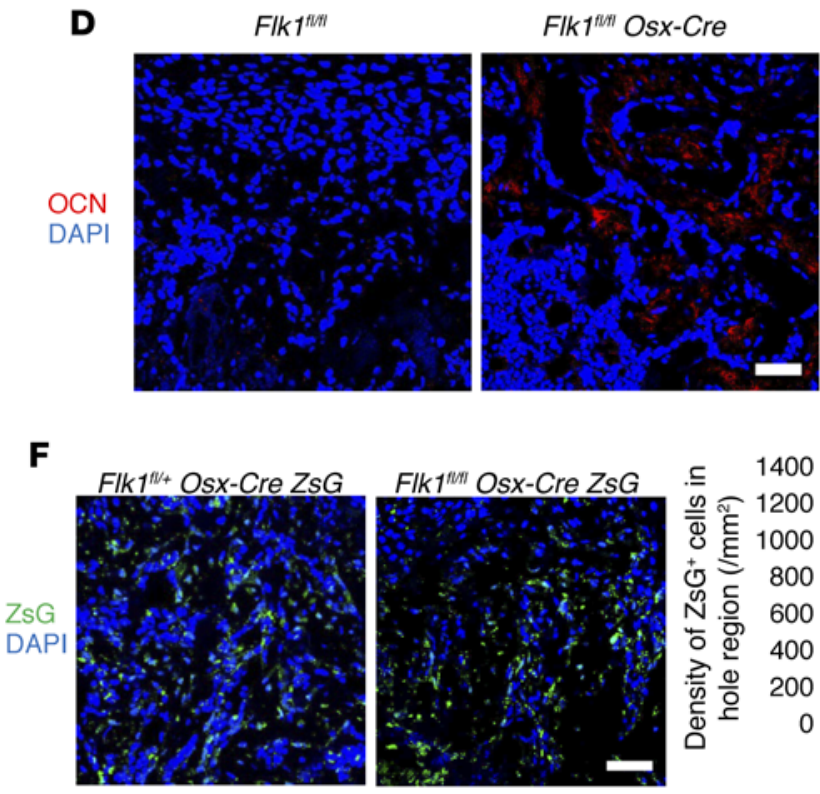

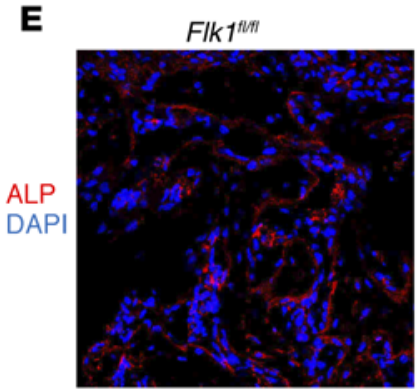

Flk $1^{\text {tul }}$ Osx-Cre

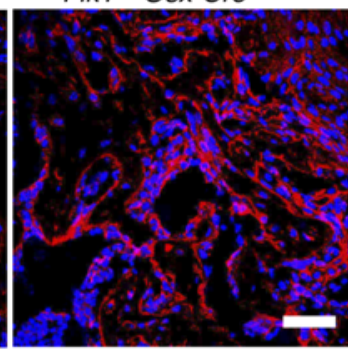

G $\mathrm{OBs}$
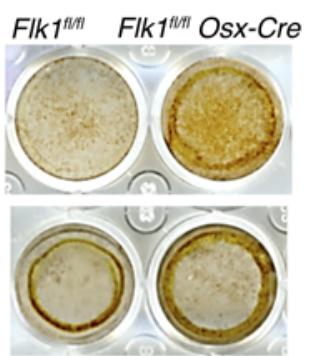

H



МС3Т3-1E Von Kossa 
Figure 9. Deletion of Vegfr2 in osteoblastic cells increases their maturation and mineralization during bone repair at PSD7. (A) 3D reconstruction of injured tibiae (top panel; scale bar: $500 \mu \mathrm{m}$ ) and mineralized bone in hole region (lower panel; scale bar: $100 \mu \mathrm{m}$ ). Representative images of 6-7 mice for each genotype. BVs in hole of Flk $\mathrm{ffl}^{\prime / f l}\left(0.0120 \pm 0.0024 \mathrm{~mm}^{3}\right)$ and Flk $7^{f / /}$ Osx -Cre mice $\left(0.0082 \pm 0.0016 \mathrm{~mm}^{3}\right)$ not statistically different, but

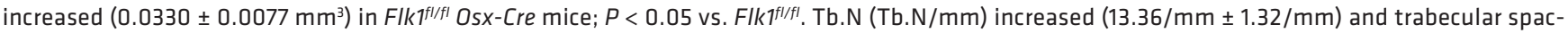
ing (Tb.Sp) decreased $(0.079 \pm 0.011 \mathrm{~mm})$, in Flk $7^{f l / f l}$ Osx-Cre compared with Flk $1^{f l / f l}$ mice $(7.08 / \mathrm{mm} \pm 0.77 / \mathrm{mm}$ and $0.160 \pm 0.023 \mathrm{~mm}) ; P<0.01(\mathrm{~Tb} . \mathrm{N})$, $P<0.05$ (Tb.Sp). (B) BV/TV of mineralized bone formed in hole and wounded marrow of control and Vegfr2-deficient mice. (C) Reduced aniline blue staining in hole region (yellow rectangles) of Flk $f^{f l+}$ Osx-Cre $(21.86 \% \pm 1.53 \%)$ compared with Flk $7^{f l f l}(36.70 \% \pm 5.47 \%)$ mice; $P<0.05$, but increased in Flk $7^{f l / f l}$ Osx-Cre (37.75\% $\left.\pm 10.99 \%\right)$ compared with Flk $7^{f l /}$ Osx-Cre mice; $P<0.05$. Right: Increased mineralization/collagen ratio in Flk $7^{f / f l}$ Osx-Cre mice. (D and E) Increased anti-OCN $(2.8 \% \pm 0.6 \%)$ and anti-ALP staining $(20.0 \% \pm 3.4 \%)$ in hole region of Flk $7^{f l / f l}$ Osx-Cre compared with Flk $7^{f l / f l}$ mice $(0.6 \% \pm 0.4 \%$ and $6.7 \% \pm 1.8 \%) ; P<0.05$ (OCN) and $P<0.01$ (ALP). (F) Similar density of ZsG ${ }^{+}$cells in hole region of Osx-Cre/ZsG, Flk $7^{f /+} \mathrm{Osx}-\mathrm{Cre} / \mathrm{ZsG}$, and Flk $7^{f / f l} \mathrm{Osx}$-Cre/ZsG mice. (C) Von Kossa staining of osteoblasts cultured for 21 days in mineralization medium with or without BMP2. (H) Von Kossa staining of MC3T3-1E cells cultured for 21 days in mineralization medium containing $100 \mu \mathrm{M}$ VEGFR2 kinase inhibitor or vehicle (Veh). Data are representative of 3 independent experiments (G and $\mathbf{H})$. Scale bars: $200 \mu \mathrm{m}$ (C), $50 \mu \mathrm{m}$ (D-F); $n=6-7$ (B-E); $n=4-6$ (F). ANOVA with Tukey's post-hoc test $(\mathbf{A}-\mathbf{C}$ and $\mathbf{F})$ and unpaired 2-tailed Student's $t$ test $(\mathbf{D}$ and $\mathbf{E})$ were used. ${ }^{*},{ }^{\#} P<0.01 ;{ }^{\# \#} P<0.05 .{ }^{*}$, vs. Flk ${ }^{f / / f l}$. \#,\#\#s. Flk $7^{f / /+}$ Osx-Cre.

previous studies showing that Vegfa transcripts in extracts of bones from Vegfa CKO mice were reduced by about $70 \%$ compared with controls (24). To allow better tracing of osteoblastic cells, ZsG, a Cre-activated and strongly fluorescent reporter, was introduced into Osx-Cre, Vegfafl/+ Osx-Cre, and Vegfa CKO mice. Vegfal/fll VE-cadherin-Cre and Flk $1^{f / f l}$ Osx-Cre mice were generated using a similar strategy.

Tibial monocortical defect model. The tibial monocortical defect model used is a simplified stable fracture model, described previously $(33,67,68)$. Skeletal mature mice (9- to 12 -week-old males) were used for all experiments. Mice were placed under general anesthesia by i.p. injection of $100 \mathrm{mg} / \mathrm{kg}$ ketamine and $10 \mathrm{mg} / \mathrm{kg}$ xylazine. The lateral aspect of right tibia was exposed and carefully cleared of overlying soft tissues while preserving the PO. A monocortical osseous hole (0.8 $\mathrm{mm}$ diameter) was created on the anterior surface of the tibia crest using a round burr attached to a dental drill. Irrigation with saline was used to remove bone dust and fragments. The soft-tissue wound was closed by separately suturing the muscle and skin layers with 5-0 absorbable gut suture (Reli). After surgery, mice received s.c. injection of $0.05-0.1 \mathrm{mg} / \mathrm{kg}$ buprenorphine for analgesia.

Delivery of recombinant VEGF. Absorbable collagen hemostatic sponges (Avitene) were cut to the dimensions of the injured region. Sponges were soaked in $1 \mu$ l of human recombinant VEGF165 (R\&D Systems) with a concentration of 1 or $0.1 \mu \mathrm{g} / \mu \mathrm{l}$ for 30 minutes at $4^{\circ} \mathrm{C}$. Control sponges were soaked in PBS. After generation of the cortical defect, the loaded sponge was inserted into the defect, followed by a single stitch of the cut muscle flap to cover the wound.

$\mu C T$ analysis. Tibiae were isolated, fixed overnight in $10 \%$ neutral buffered formalin, and kept in $70 \%$ ethanol until analyzed using a $\mu$ CT35 system (SCANCO Medical) with a spatial resolution of 7 $\mu \mathrm{m}$. Sagittal image sections of injured tibiae were used to perform 3D histomorphometric analysis. We defined the regions of interest as (i) the hole region between the interrupted cortical bone ends, (ii) injured BM, and (iii) periosteal callus outside the hole. Old bone fragments remaining from the drilling were excluded from the regions of interest. A total of 96-100 consecutive images (about 0.672-0.7 mm in length) were used for $3 \mathrm{D}$ reconstruction and analysis, covering most of the injured region and periosteal callus. Structural parameters (3D) included total tissue volume (TV), trabecular BV per TV (BV/TV), Tb.N, Tb.Th, and Tb.Sp.

Histology and histomorphometry. Fixed samples were decalcified in 0.5 M EDTA ( $\mathrm{pH}$ 8.0) for 14 days and embedded in paraffin or optimal cutting temperature (OCT) compound (Tissue-Tek). Longitudinal sections $(8 \mu \mathrm{m})$ using the hole region as landmark were cut and stained for H\&E, aniline blue, TRAP, movat pentachrome, or Safranin O. Comparisons between Vegfa CKO and control mice were primarily covering the hole region between the interrupted cortical bone ends and adjacent areas, visible in the same sections. In most cases, staining in the wounded BM was not quantified because histological details in longitudinal sections of BM could not be guaranteed to be in the same horizontal planes as those of the hole regions.

Six to 8 tissue sections for each mouse, stained with aniline blue, were used to determine the amount of newly formed osseous tissue. Each section was photographed and analyzed using a published method (67). The digital images were imported into Adobe Photoshop CS5. A rectangular region of interest covering the hole region encompassed about $10^{6}$ pixels. The number of aniline blue-stained pixels was determined using the magic wand tool. The relative amount of collagen in the hole region was calculated as aniline blue-positive pixels $/ 10^{6}$ pixels. The mineralization/collagen ratio was calculated as BV/TV $(\mu \mathrm{CT})$ divided by the percent of aniline blue-stained area. For quantifying the amount of cartilage, a periosteal area about $2 \mathrm{~mm}$ distal to the hole defect was selected, and Safranin O-positive areas were determined using the magic wand tool.

Immunofluorescence and IHC. Immunostaining was performed using a standard protocol. Sections were incubated with primary antibody against neutrophil marker NIMP-R14 (1:100; Santa Cruz Biotechnology Inc., catalog sc-59338), macrophage marker F4/80 (1:100; AbD Serotek, catalog MCA497GA), CD31 (1:50, BD Biosciences, catalog 550274), CD45R (1:100, BD Biosciences, catalog 550286), Osx (1:50, Santa Cruz Biotechnology Inc., catalog sc-22538), BSP (1:50, Santa Cruz Biotechnology Inc., catalog sc-292394), OCN (1:50, Santa Cruz Biotechnology Inc., catalog sc-18322), ALP (1:50, Santa Cruz Biotechnology Inc., catalog sc-30203), VEGF (1:50, Santa Cruz Biotechnology Inc., catalog sc-152), BMP2 (1:100, Abcam, catalog ab14933), SOX9 (1:50, Santa Cruz Biotechnology Inc., catalog sc-20095), collagen type X (1:500, Calbiochem, catalog 234196), FSP1 (1:100, Abcam, catalog ab27957), or with biotin-conjugated lectin (1:100, Vector Laboratories, catalog B1205), overnight at $4^{\circ} \mathrm{C}$. For IHC, horseradish peroxidase-streptavidin $(\mathrm{ABC})$ was used to detect immunoactivity, followed by counterstaining with hematoxylin. For immunofluorescence, secondary fluorescent antibodies (1:200, Invitrogen) were added and slides were incubated at room temperature for 90 minutes in the dark. Sections were stained with or without DAPI and coverslipped as above. ImageJ was used for the quantification of BSP, OCN, VEGF, and ALP levels. Blood vessel (microvessel) 
density was calculated based on anti-CD31 staining. Single CD31 immunoreactive endothelial cells, or endothelial cell clusters separate from other microvessels, were counted as individual vessels. The total number of blood vessels in a specific field divided by total area was considered as blood vessel density. For BrdU staining, mice were injected with biotin-conjugated BrdU $(20 \mathrm{mg} / \mathrm{kg}$, SigmaAldrich) 3 hours prior to euthanasia. A commercial BrdU staining kit (Invitrogen) was used to stain proliferative cells. For detection of apoptotic cells, an in situ cell death detection kit, TMR Red (Roche Diagnostics), was used as described in manufacturer's instructions.

Isolation of osteoblastic cells from mouse long bones. Methods for isolation and culture of primary osteoblasts from hind limbs of 8- to 12-week-old mice were as described (69). BM cells were flushed out, and marrow-free diaphyses were cut into small pieces and incubated in $1 \mathrm{mg} / \mathrm{ml}$ collagenase II (Invitrogen) at $37^{\circ} \mathrm{C}$ for 1 hour with constant shaking. The bone pieces were cultured in DMEM containing 15\% FBS (HyClone) and $100 \mu \mathrm{g} / \mathrm{ml}$ ascorbic acid (Sigma-Aldrich) until cells migrating from the bone chips became confluent.

Isolation of BMSCs. BM cells from hind limbs of 8- to 12-week-old mice were flushed out and cultured in $\alpha$-MEM containing $10 \%$ FBS. The cultures were kept undisturbed until day 4 , when half of the medium was changed. This was followed by change of medium every other day until day 14. Cells were passaged and maintained in complete culture medium. Cells in passages 1-2 of these BMSCs were used for differentiation assays and passages $2-4$ for migration and proliferation assays.

Adenoviral infection. Adenoviruses were purchased from Vector BioLabs. We cultured BMSCs in 12-well plates with $\alpha$-MEM medium containing 10\% FBS for 48 hours before they reached confluence, exposed them to adenoviral-Cre or adenoviral GFP $\left(2 \times 10^{7}\right.$ plaqueforming units per well) for 48 hours, and continued the culture in differentiation medium.

In vitro osteoblastic differentiation and mineralization assay. Murine osteoblastic MC3T3-E1 (subclone 4) cells were purchase from ATCC and cultured in ascorbic acid-free $\alpha$-MEM containing $10 \%$ FBS. Cells of passages 6-7 were used for the mineralization assay. MC3T3-E1 cells, BMSCs, and osteoblastic cells from long bones were seeded in 12-well or 24-well plates containing complete culture medium. Upon reaching 70\%-80\% confluency, cells were switched to mineralization medium (10\% FBS, $10 \mathrm{mM} \beta$-glycerophosphate, and $50 \mu \mathrm{g} / \mathrm{ml}$ ascorbic acid) added with or without $100 \mathrm{nM}$ dexamethasone or $50 \mathrm{ng} / \mathrm{ml}$ BMP2. To study effects of exogenous VEGF on mineralization of BMSCs and MC3T3-1E cells, 10 or $40 \mathrm{ng} / \mathrm{ml}$ recombinant VEGF (R\&D Systems) was added to the mineralization medium. To test the role of VEGFR2 in mineralization of MC3T3-1E cells, $100 \mu \mathrm{M}$ VEGFR2 kinase inhibitor I (EMD Millipore, catalog $676480)$ was added to the mineralization medium. The mineraliza- tion medium was replaced every 2 days. After 21 days of culture, Von Kossa or Alizarin red staining was used to measure mineralization.

In vitro wound-closure assay. Confluent monolayers of BMSCs (passages 2-4) were scratched with a yellow pipette tip to create an about $1,000-\mu \mathrm{m}$ wide cell-free wound. Wounded monolayers were incubated for 24 hours and photographed at time 0 and at 24 hours. The average rate of wound closure was calculated as the difference between the starting width and the width at any incubation time/incubated time.

Transwell migration assay. Transwell chambers (Costar, Corning Inc.) with a polycarbonate membrane $(6.5 \mathrm{~mm}$ diameter, $8 \mu \mathrm{m}$ pore size) were used to study the migration of BMSCs. The lower chamber contained complete culture medium. BMSCs were serumstarved for 12 hours before harvest. Cells $\left(0.8 \times 10^{5}\right)$ were seeded in the upper chambers in serum-free medium containing $0.2 \%$ BSA (Sigma-Aldrich). After 14 hours incubation at $37^{\circ} \mathrm{C}$, cells on the top surface of the membrane were wiped off, and the membranes were fixed and stained with $0.1 \%$ crystal violet. Cells remaining on the bottom surface of the membrane were examined under a microscope. Cells from 6-10 random fields across 3 replicate wells were captured for counting, and the average number of invaded cells per field represented the migration activities.

Statistics. The data are presented as mean \pm SEM using unpaired 2-tailed Student's $t$ test or ANOVA with Tukey's post-hoc test for multiple comparisons. Spearman's correlation coefficient was used to measure the dependency of 2 variables. $P$ values less than 0.05 were considered significant.

Study approval. All animal experiments were approved by the Harvard Medical Area Standing Committee on Animals and in accordance with the US Public Health Service Policy on Humane Care and Use of Laboratory Animals.

\section{Author contributions}

KH designed studies, conducted experiments, acquired and analyzed data, and wrote the manuscript. BRO designed studies, analyzed data, and wrote the manuscript.

\section{Acknowledgments}

Genentech provided mice with floxed Vegfa and Flk1 alleles. We thank Yulia Pittel for secretarial assistance and Nikon Imaging Center at Harvard Medical School for help with light and fluorescence microscopy. This work was supported by NIH grant AR36819 (to B.R. Olsen).

Address correspondence to: Bjorn R. Olsen, Harvard School of Dental Medicine, 188 Longwood Ave., Boston, Massachusetts, USA. Phone: 617.432.1874; E-mail: bjorn_olsen@hms.harvard.edu.
1. Gomez-Barrena E, Rosset P, Lozano D, Stanovici J, Ermthaller C, Gerbhard F. Bone fracture healing: Cell therapy in delayed unions and nonunions. Bone. 2015;70:93-101.

2. Marsell R, Einhorn TA. The biology of fracture healing. Injury. 2011;42(6):551-555.

3. Claes L, Recknagel S, Ignatius A. Fracture healing under healthy and inflammatory conditions. Nat Rev Rheumatol. 2012;8(3):133-143.

4. Dimitriou R, Tsiridis E, Giannoudis PV. Current concepts of molecular aspects of bone healing.
Injury. 2005;36(12):1392-1404.

5. Coultas L, Chawengsaksophak K, Rossant J. Endothelial cells and VEGF in vascular development. Nature. 2005;438(7070):937-945.

6. Hoeben A, Landuyt B, Highley MS, Wildiers H, Van Oosterom AT, De Bruijn EA. Vascular endothelial growth factor and angiogenesis. Pharmacol Rev. 2004;56(4):549-580.

7. Gerber HP, Vu TH, Ryan AM, Kowalski J, Werb Z, Ferrara N. VEGF couples hypertrophic cartilage remodeling, ossification and angiogenesis during endochondral bone formation. Nat Med. 1999;5(6):623-628

8. Zelzer E, Mamluk R, Ferrara N, Johnson RS, Schipani E, Olsen BR. VEGFA is necessary for chondrocyte survival during bone development. Development. 2004;131(9):2161-2171.

9. Street J, Winter D, Wang JH, Wakai A, McGuinness A, Redmond HP. Is human fracture hematoma inherently angiogenic? Clin Orthop Relat R. 2000;378(378):224-237.

10. Leek RD, Hunt NC, Landers RJ, Lewis CE, Royds 
JA, Harris AL. Macrophage infiltration is associated with VEGF and EGFR expression in breast cancer. J Pathol. 2000;190(4):430-436.

11. Barleon B, Sozzani S, Zhou D, Weich HA, Mantovani A, Marme D. Migration of human monocytes in response to vascular endothelial growth factor (VEGF) is mediated via the VEGF receptor flt-1. Blood. 1996;87(8):3336-3343.

12. Tarkka T, et al. Adenoviral VEGF-A gene transfer induces angiogenesis and promotes bone formation in healing osseous tissues. J Gene Med. 2003;5(7):560-566.

13. Peng HR, et al. Synergistic enhancement of bone formation and healing by stem cell-expressed VEGF and bone morphogenetic protein-4. J Clin Invest. 2002;110(6):751-759.

14. Street J, et al. Vascular endothelial growth factor stimulates bone repair by promoting angiogenesis and bone turnover. Proc Natl Acad Sci U S A. 2002;99(15):9656-9661.

15. Jacobsen KA, et al. Bone formation during distraction osteogenesis is dependent on both VEGFR1 and VEGFR2 signaling. J Bone Miner Res. 2008;23(5):596-609.

16. Behr B, Leucht P, Longaker MT, Quarto N. Fgf-9 is required for angiogenesis and osteogenesis in long bone repair. Proc Natl Acad Sci U S A. 2010;107(26):11853-11858.

17. Emad B, Sherif el M, Basma GM, Wong RW, Bendeus M, Rabie AB. Vascular endothelial growth factor augments the healing of demineralized bone matrix grafts. Int J Surg. 2006;4(3):160-166.

18. Aldridge SE, Lennard TWJ, Williams JR, Birch MA. Vascular endothelial growth factor receptors in osteoclast differentiation and function. Biochem Biophys Res Commun. 2005;335(3):793-798.

19. Niida S, et al. Vascular endothelial growth factor can substitute for macrophage colony-stimulating factor in the support of osteoclastic bone resorption. JExp Med.1999;190(2):293-298.

20. Wang DS, et al. Increase of vascular endothelial growth factor mRNA expression by $1,25-$ dihydroxyvitamin D-3 in human osteoblastlike cells. J Bone Miner Res. 1996;11(4):472-479.

21. Zelzer E, Olsen BR. Multiple roles of vascular endothelial growth factor (VEGF) in skeletal development, growth, and repair. Curr Top Dev Biol. 2005;65:169-187.

22. Maes C, et al. Osteoblast precursors, but not mature osteoblasts, move into developing and fractured bones along with invading blood vessels. Dev Cell. 2010;19(2):329-344.

23. Zelzer E, et al. Skeletal defects in VEGF(120/120) mice reveal multiple roles for VEGF in skeletogenesis. Development. 2002;129(8):1893-1904

24. Liu YQ, et al. Intracellular VEGF regulates the balance between osteoblast and adipocyte differentiation. J Clin Invest. 2012;122(9):3101-3113.

25. Wang Y, et al. The hypoxia-inducible factor a pathway couples angiogenesis to osteogenesis during skeletal development. J Clin Invest. 2007;117(6):1616-1626.

26. Swift ME, Kleinman HK, DiPietro LA. Impaired wound repair and delayed angiogenesis in aged mice. Lab Invest. 1999;79(12):1479-1487.

27. Jiang S, Haider HK, Ahmed RPH, Idris NM, Salim A, Ashraf M. Transcriptional profiling of young and old mesenchymal stem cells in response to oxygen deprivation and reparability of the infarcted myocardium. J Mol Cell Cardiol. 2008;44(3):582-596.

28. Pola R, et al. Age-dependent VEGF expression and intraneural neovascularization during regeneration of peripheral nerves. Neurobiol Aging. 2004;25(10):1361-1368.

29. Wilson A, Shehadeh LA, Yu H, Webster KA. Age-related molecular genetic changes of murine bone marrow mesenchymal stem cells. $B M C$ Genomics. 2010;11:229.

30. Efimenko A, Starostina E, Kalinina N, Stolzing A. Angiogenic properties of aged adipose derived mesenchymal stem cells after hypoxic conditioning. J Transl Med. 2011;9:10.

31. Costa N, Paramanathan S, Mac Donald D, Wierzbicki AS, Hampson G. Factors regulating circulating vascular endothelial growth factor (VEGF): Association with bone mineral density (BMD) in post-menopausal osteoporosis. Cytokine. 2009;46(3):376-381.

32. Maharaj AS, D'Amore PA. Roles for VEGF in the adult. Microvasc Res. 2007;74(2-3):100-113.

33. Kim JB, et al. Bone regeneration is regulated by Wnt signaling. J Bone Miner Res. 2007;22(12):1913-1923.

34. Kawao N, et al. Plasminogen Plays a Crucial Role in Bone Repair. J Bone Miner Res. 2013;28(7):1561-1574.

35. He LZ, Marneros AG. Macrophages are essential for the early wound healing response and the formation of a fibrovascular scar. Am J Pathol. 2013;182(6):2407-2417.

36. Lin EY, et al. Macrophages regulate the angiogenic switch in a mouse model of breast cancer. Cancer Res. 2006;66(23):11238-11246.

37. Clauss $\mathrm{M}$, et al. Vascular permeability factor: a tumor-derived polypeptide that induces endothelial cell and monocyte procoagulant activity, and promotes monocyte migration. J Exp Med. 1990;172(6):1535-1545

38. Sunderkotter C, Goebeler M, Schulzeosthoff K, Bhardwaj R, Sorg C. Macrophage-derived angiogenesis factors. Pharmacol Ther. 1991;51(2):195-216.

39. Tonnesen MG, Feng XD, Clark RA. Angiogenesis in wound healing. J Investig Dermatol Symp Proc. 2000;5(1):40-46.

40. Wu AC, Raggatt LJ, Alexander KA, Pettit AR. Unraveling macrophage contributions to bone repair. Bonekey Rep. 2013;2:373.

41. Wang CJ, et al. VEGF modulates angiogenesis and osteogenesis in shockwavepromoted fracture healing in rabbits. J Surg Res. 2011;171(1):114-119.

42. Yamauchi K, et al. Vascular endothelial cell growth factor attenuates actions of transforming growth factor- $\beta$ in human endothelial cells. J Biol Chem. 2004;279(53):55104-55108.

43. Pennock S, Kazlauskas A. Vascular Endothelial Growth Factor A Competitively Inhibits Platelet Derived Growth Factor (PDGF)-Dependent Activation of PDGF Receptor and Subsequent Signaling Events and Cellular Responses. Mol Cell Biol. 2012;32(10):1955-1966.

44. Greenberg JI, et al. A role for VEGF as a negative regulator of pericyte function and vessel maturation. Nature. 2008;456(7223):809-813.
45. Caplan AI, Correa D. PDGF in bone formation and regeneration: new insights into a novel mechanism involving MSCs. JOrthop Res. 2011;29(12):1795-1803.

46. Kaipel M, et al. BMP-2 but not VEGF or PDGF in fibrin matrix supports bone healing in a delayed-union rat model. JOrthop Res. 2012;30(10):1563-1569.

47. Schonmeyr BH, Soares M, Avraham T, Clavin NW, Gewalli F, Mehrara BJ. Vascular endothelia growth factor inhibits bone morphogenetic protein 2 expression in rat mesenchymal stem cells. Tissue Eng Pt A. 2010;16(2):653-662.

48. Eckardt H, Bundgaard KG, Christensen KS, Lind M, Hansen ES, Hvid I. Effects of locally applied vascular endothelial growth factor (VEGF) and VEGF-inhibitor to the rabbit tibia during distraction osteogenesis. JOrthop Res. 2003;21(2):335-340.

49. Geng HQ, Song H, Qi J, Cui DX. Sustained release of VEGF from PLGA nanoparticles embedded thermo-sensitive hydrogel in full-thickness porcine bladder acellular matrix. Nanoscale Res Lett. 2011;6(1):312.

50. Silva EA, Mooney DJ. Effects of VEGF temporal and spatial presentation on angiogenesis. Biomaterials. 2010;31(6):1235-1241.

51. Gerber HP, et al. VEGF regulates haematopoietic stem cell survival by an internal autocrine loop mechanism. Nature. 2002;417(6892):954-958.

52. Lee $\mathrm{S}$, et al. Autocrine VEGF signaling is required for vascular homeostasis. Cell. 2007;130(4):691-703.

53. Chan CKF, et al. Identification and specification of the mouse skeletal stem cell. Cell. 2015;160(1-2):285-298.

54 . Kubo $S$, et al. Blocking vascular endothelial growth factor with soluble Flt-1 improves the chondrogenic potential of mouse skeletal muscle-derived stem cells. Arthritis Rheum. 2009;60(1):155-165.

55. Matsumoto $\mathrm{T}$, et al. Cartilage repair in a rat model of osteoarthritis through intraarticular transplantation of muscle-derived stem cells expressing bone morphogenetic protein 4 and soluble Flt-1. Arthritis Rheum. 2009;60(5):1390-1405.

56 . Tang Y, et al. TGF- $\beta 1$-induced migration of bone mesenchymal stem cells couples bone resorption with formation. Nat Med. 2009;15(7):757-765.

57. Crane JL, Cao X. Function of matrix IGF-1 in coupling bone resorption and formation. J Mol Med. 2014;92(2):107-115.

58. Xie H, et al. PDGF-BB secreted by preosteoclasts induces angiogenesis during coupling with osteogenesis. Nat Med. 2014;20(11):1270-1278.

59. Chung YS, et al. Association of vascular endothelial growth factor gene polymorphisms with osteoporotic vertebral compression fractures in postmenopausal women. Genes Genom. 2010;32(6):499-505.

60. Kyllonen L, D’Este M, Alini M, Eglin D. Local drug delivery for enhancing fracture healing in osteoporotic bone. Acta Biomater. 2015;11:412-434.

61. Peng HR, et al. VEGF improves, whereas sFlt1 inhibits, BMP2-induced bone formation and bone healing through modulation of angiogenesis. J Bone Miner Res. 2005;20(11):2017-2027.

62. Mundy G, et al. Stimulation of bone forma- 


\section{RESEARCH ARTICLE}

tion in vitro and in rodents by statins. Science. 1999;286(5446):1946-1949.

63. Shah SR, Werlang CA, Kasper FK, Mikos AG. Novel applications of statins for bone regeneration. Natl Sci Rev. 2015;2(1):85-99.

64. Tikiz C, Tikiz H, Taneli F, Gumuser G, Tuzun C. Effects of simvastatin on bone mineral density and remodeling parameters in postmenopausal osteopenic subjects: 1-year follow-up study. Clin
Rheumatol. 2005;24(5):447-452.

65. Rodda SJ, McMahon AP. Distinct roles for Hedgehog and canonical Wnt signaling in specification, differentiation and maintenance of osteoblast progenitors. Development. 2006;133(16):3231-3244.

66. Alva JA, et al. VE-Cadherin-Cre-recombinase transgenic mouse: a tool for lineage analysis and gene deletion in endothelial cells. Dev Dyn. 2006;235(3):759-767.
The Journal of Clinical Investigation

67. Minear S, et al. Wnt proteins promote bone regeneration. Sci Transl Med.2010;2(29):29ra20.

68. Minear S, Leucht P, Miller S, Helms JA. rBMP represses Wnt signaling and influences skeletal progenitor cell fate specification during bone repair. J Bone Miner Res. 2010;25(6):1196-1207.

69. Bakker A, Klein-Nulend J. Osteoblast isolation from murine calvariae and long bones. Methods MolMed.2003;80:19-28. 